Citation: S. De Falco (2020) Un modello basato sulla teoria dei grafi per l'individuazione di isomorfismi distrettuali geograficamente non prossimi. II caso dei distretti industriali italiani. Bollettino della Società Geografica Italiana serie 14, 3(1): 27-50. doi: 10.36253/bsgi. v3i1.898

Copyright: (c) 2020 S. De Falco This is an open access, peer-reviewed article published by Firenze University Press (http://www.fupress.com/bsgi) and distributed under the terms of the Creative Commons Attribution License, which permits unrestricted use, distribution, and reproduction in any medium, provided the original author and source are credited.

Data Availability Statement: All relevant data are within the paper and its Supporting Information files.

Competing Interests: The Author(s) declare(s) no conflict of interest.

\section{Un modello basato sulla teoria dei grafi per l'individuazione di isomorfismi distrettuali geograficamente non prossimi. Il caso dei distretti industriali italiani}

\author{
A graph theory based model for the identification of \\ geographically non-close district isomorphisms. The case of the \\ Italian industrial districts
}

\author{
Stefano De Falco \\ Dipartimento di Scienze Politiche, Università degli Studi di Napoli Federico II, Italia \\ E-mail: sdefalco@unina.it
}

\begin{abstract}
A dichotomy often frequent in the context of geographic studies concerns the dualism between propagation and induction models-based phenomena, inherent in variables and factors characterizing contiguous areas, and research relating to homogeneity between geographically not closed areas. In the wake of the latter research, this contribution proposes a model that exploits the potential of graph theory for the evaluation of common dynamics relating to non-contiguous areas. The assumption underlying the model envisages configuring the reality being studied in terms of a network whose nodes and branches are respectively representative of entities distant from each other and of their related affinities. The proposed approach focuses on some Italian industrial districts. The value of the proposed approach is twofold, on the one hand regarding the specific industrial district topic with both scientific and practical implications, and on the other hand it aims to provide a method that can be replicated in similar scenarios in which it is interesting to evaluate the similarity between neighboring areas analytically.
\end{abstract}

Keywords: Industrial districts, Graphs Theory, network analysis, isomorphisms, cluster.

Riassunto. Una dicotomia spesso frequente nell'ambito degli studi geografici riguarda il dualismo tra fenomeni basati su modelli di propagazione e induzione inerenti variabili e fattori caratterizzanti aree contigue, e ricerche relative a omogeneità tra aree geograficamente non prossime. Nel solco di quest'ultimo filone, il presente contributo propone un modello che sfrutta la potenzialità della teoria dei grafi per la valutazione di dinamiche comuni relative ad aree non contigue. Lassunzione alla base del modello prevede di configurare la realtà oggetto di studio in termini di una rete i cui nodi e rami risultino rispettivamente rappresentativi di entità distanti tra loro e di eventuali relative affinità.Il caso applicativo nel quale è declinata la metodologia è relativo ad alcuni distretti industriali italiani. La valenza dell'approccio proposto è di duplice natura, riguardando da un lato lo specifico ambito distrettuale sia con implicazioni scientifiche che pratiche, dall'altro la trasponibilità del metodo ad ambiti differenti nei quali 
interessi valutare dinamiche comuni tra aree geograficamente non prossime.

Parole chiave: Distretti industriali, Teoria dei grafi, analisi di rete, isomorfismi, cluster.

\section{Introduzione}

Il particolare interesse geografico-economico per i distretti industriali è dato, fin dalla primigenia elaborazione marshalliana, dalle economie esterne (Tinacci Mossello, 1982).

Marshall "non trascura certamente le economie nella produzione che derivano dalle risorse, dall'organizzazione interna, e dall'efficienza nella gestione dell'impresa (economie interne)", ma "nel contempo introduce una seconda fonte di economie che dipendono dalle relazioni sociali e di produzione che si formano al di fuori dello stabilimento, ma all'interno del territorio che lo comprende" (Conti 2012, 275), appunto le economie esterne.

Negli anni Settanta Becattini e Brusco (Becattini 1975; Brusco 1975) iniziano a delineare i tratti distintivi di uno sviluppo industriale italiano che, a fronte di una corrispondente perdita di occupazione e di un declino irrecuperabile nelle grandi imprese, risulta essere caratterizzato dalla proliferazione relativamente autonoma delle piccole e medie imprese - "grappoli di piccole imprese in stretta relazione con il contesto socioeconomico" (Conti 2012, 275). L'inizio del mutamento di prospettiva teorica, rispetto ad un già consolidato e più "classico" modello interpretativo corrente dello sviluppo industriale italiano, in cui un sottosviluppato Sud si contrapponeva ad un Nord intensamente industrializzato e principalmente sostenuto da una costellazione di grandi imprese attorno alle quali gravitavano unità medie o piccole da esse dipendenti, si è avuto in Italia solo nel momento in cui l'evidenza empirica ricavata dai dati del censimento del 1971 (disponibili dopo il 1974) andava a confermare in maniera innegabile le letture dei fenomeni di sviluppo industriale della Toscana in primo luogo, e di altri importanti lembi del territorio nazionale poi (Falorni 2013).

Brusco $(1989,461)$ riferisce, infatti, che il primo scritto di Becattini nel quale "per la prima volta nella letteratura economica italiana del dopoguerra, sosteneva che i sistemi di piccole imprese potevano essere vitali e competitivi e che era sbagliato interpretarli soltanto come resti di un sistema produttivo condannato alla sconfitta" è del 1969, pubblicato in un volume dell'IR-
PET (Istituto Regionale per la Programmazione Economica della Toscana).

Nei successivi anni Ottanta si celebra il successo del modello distrettuale italiano e del "piccolo è bello", in ragione delle capacità di resistenza e sviluppo date dalle flessibili PMI rispetto alle rigide GI fordiste, che collasseranno. È degli anni Novanta, invece, la crisi generalizzata delle strutture distrettuali, non casualmente coincidente con il decollo della globalizzazione

Sebbene quindi la roadmap dei distretti non risulti caratterizzata da una dinamica lineare, il dibattito scientifico che li ha riguardati è stato dagli anni '90 ad oggi sempre molto acceso e ricco di contributi che fanno ritenere l'interesse scientifico verso tale forma organizzativa industriale molto rilevante e ancora sempre attuale. Per rendersene conto basta guardare solo ad alcuni esempi di lavori pubblicati nel 2020, specificamente sui distretti industriali italiani (Mariotti et al. 2020; Festa et al. 2020; Colavitti, Usai 2020; Simboli et al. 2020); inoltre uno spunto in tal senso interessante e dimostrativo della attualità scientifica di tale paradigma può essere ritrovato in un recente lavoro di Cortés ed altri autori (Cortés et al. 2019) nel quale è presentato uno studio dal 1990 fino al 2017 di disamina della corposa e sempre crescente letteratura sull'argomento distrettuale.

Tale copiosa letteratura di settore comprende diverse prospettive di indagine scientifica, da quella economica, sociologica, tecnologica fino ovviamente a quella geografica, riscontrabile ad esempio nei lavori di Maria Tinacci Mossello (1990), Fabio Sforzi (2008; 2014), Sergio Conti (2012) e in quelli raccolti nello speciale n. 2 della rivista Geotema del 1995, relativi agli autori Taylor, Dini, Tödtling e Bonavero.

Il contesto geografico rappresenta, infatti, un fattore incidente sia per la competitività della singola azienda, sia - soprattutto - per la forma agglomerata di imprese in un distretto, costituendo quello che Camison e Molina-Morales (1998) definiscono l'effetto del territorio.

Finora l'approccio al tema distrettuale è stato prevalentemente incentrato sulla opportunità di indagare esclusivamente il rapporto tra contesto geografico e singolo distretto, analizzandone l'effetto del particolare territorio $x$ in relazione al distretto $y$ ivi insediato. La visione sistemica dell'insieme dei distretti è stata, invece, presente solo nella forma di report e banche dati prive di particolari elaborazioni comparative atte a rilevare elementi scientifici di interesse geografico.

Sulla base di tale evidenza, nella presente ricerca si intende proporre un modello che consenta di considerare il sistema-distretti nella sua interezza a livello Paese. La finalità di tale scelta si concretizza nella opportunità di individuare, all'intero di un tale sistema, eventuali 
cluster omogenei di distretti sulla base di affinità delle loro principali variabili caratterizzanti. Lapproccio basato sulle metriche per l'analisi di rete consente, infatti, di identificare tra i vari possibili scenari di sottoinsiemi distrettuali quelli che presentano maggiori livelli di affinità. Per tale motivo nella ricerca si è voluto anche dal punto di vista semantico battezzare tali ipotesi di scenario come isomorfismi distrettuali ${ }^{1}$. Trattandosi di distretti scelti su scala nazionale ed eventualmente aggregati da variabili di valori simili e, dunque, non sulla base di una contiguità territoriale, si è deciso di definirli isomorfismi distrettuali geograficamente non prossimi.

La valenza dell'approccio risiede nel fatto che, a partire dalla informazione relativa ai cluster affini di distretti configurati sotto forma di reti i cui nodi risultano essere i distretti stessi e i cui rami risultano essere le relazioni di affinità tra le loro relative variabili caratterizzanti, è ipotizzabile il perseguimento di strategie comuni per il rafforzamento dei comuni punti di forza e per la riduzione di quelli di debolezza. In tal modo, anche in presenza di differenti effetti dei territori in contesti geograficamente non prossimi, risulta possibile ravvisare una omogeneità di esternalità, note con accezioni diverse a seconda degli autori, ad esempio "effetto cluster" (Bell 2005), "economie distrettuali esterne" (Fuensanta 2010) o "effetto di prossimità" (Puig, Marques 2011).

La base dati di ingresso a partire dalla quale sono state fatte tutte le elaborazioni è costituita dal IV Rapporto dell'Osservatorio Nazionale Distretti di Unioncamere $^{2}$. Il rapporto considerato relativo all'ultima versione rilasciata analizza una enorme mole di dati nella finestra temporale 2008-2013, che risulta essere uno scenario consolidato e meno soggetto a errori di bias, offset e aliasing tipici dei campionamenti di processi ancora in corso o da poco esauriti.

In ragione del fatto che sussistono convergenze relative ad alcune variabili distrettuali tra aree non contigue, invece di impiegare i metodi dell'autocorrelazione

\footnotetext{
${ }^{1}$ In matematica, in particolare in algebra astratta, un isomorfismo è un`applicazione biunivoca fra oggetti matematici tale che l applicazione e la sua inversa conservino le operazioni in esse definite. Intuitivamente, un isomorfismo può essere definito con le parole del matematico Douglas Hofstadter (1979): "Si parla di isomorfismo quando due strutture complesse si possono applicare l'una sull'altra, cioè far corrispondere l'una all'altra, in modo tale che per ogni parte di una delle strutture ci sia una parte corrispondente nell'altra struttura; in questo contesto diciamo che due parti sono corrispondenti se hanno un ruolo simile nelle rispettive strutture".

${ }^{2}$ L'Osservatorio nazionale distretti italiani (OND) è una banca dati sui distretti italiani realizzata nel 2009 dalla Federazione dei Distretti Italiani e dall'Unioncamere. Successivamente sono state curate altre 4 edizioni tra il 2010 e il 2014 approfondendo caratteristiche e dinamiche dei distretti industriali.
}

spaziale LISA (Local Indicator of Spatial Autocorrelation) - ad esempio impiegati da Randelli ed altri autori (2017) nella costruzione di una cluster map basata sulla prossimità geografica relativa ad aziende agricole, oppure da Las Casas et al. (2009) per l'analisi dei cluster dei flussi migratori in Italia - quale metodologia analitica si è scelto di riferirsi all'analisi di rete per la ricerca di opportune aggregazioni. In particolare, mediante il software Ucinet ${ }^{\oplus}$ sono stati identificati i diversi cluster corrispondenti a geografie a geometrie variabili in ordine agli isomorfismi via via rilevati nel corso dell'analisi corroborata da cartografie e schemi di rete.

Nello svolgimento dell'indagine sono state messe in evidenza anche condizioni di piena coincidenza tra $\mathrm{i}$ valori delle variabili distrettuali. In particolare, laddove due distretti condividevano gli stessi valori di almeno una delle loro variabili caratterizzanti ci si è riferiti ad un isomorfismo definito "perfetto".

\section{I distretti italiani nella geografia della impresa}

I dati dell'Istat dell'ultimo censimento 2011 confermano che i distretti industriali rappresentano circa un quarto del sistema produttivo italiano, in termini di lavoratori (24,5\% del totale) e unità produttive locali (24,4\% del totale). Dal punto di vista settoriale, il settore manifatturiero italiano risulta essere fortemente caratterizzato dal modello distrettuale e i distretti dell'industria manifatturiera concorrono per oltre un terzo dell'intera produzione italiana, assorbendo il 65,8\% dei lavoratori dell'industria manifatturiera (Istat 2015).

La natura e l'entità del fenomeno distrettuale italiano può essere compresa a partire dall'analisi del surplus commerciale che premia la specializzazione manifatturiera distrettuale nazionale. L'Italia è, infatti, il quinto Paese al mondo (secondo in Europa dietro alla Germania) per surplus commerciale, ad esclusione dell'energia in maggior parte importata dalla Francia. A differenza della Germania che si caratterizza per grandi imprese, il tessuto italiano risulta costituito prevalentemente, detenendo un primato europeo, da piccole e micro imprese - le famose "imprese bonsai"3 - che esprimono un forte dinamismo industriale che viene ad essere amplificato nella agglomerazione distrettuale.

Secondo Banca Intesa la crescita del fatturato delle imprese nella finestra temporale 2008-2017 è di cinque punti percentuali superiore a quella delle aree non distrettuali.

${ }^{3}$ Come le definì De Benedetti in una intervista sul New York Times nel 1992 
Certamente sono ravvisabili dei gradienti geografici che differenziano le performance distrettuali (Belussi 2015) secondo evidenze che in molti casi provengono dal passato nel quale alcune regioni, ad esempio la Toscana, si erano poste come soggetti pionieri della sperimentazione di forme agglomerate e condivise del lavoro. L'industrializzazione diffusa in Toscana (Dini, Maione 2001 e Dini, Tinacci 2011) inizia a diventare realmente visibile dopo la metà degli anni Cinquanta. Qualcosa del genere avviene anche in Emilia-Romagna, peraltro più avanti della Toscana come processo di industrializzazione. Un processo del genere in Veneto e Marche partirà solo nel decennio successivo.

Giacomo Becattini svolse ricerche sull'industrializzazione proprio della Toscana durante i primi decenni seguenti la Seconda Guerra Mondiale facendo uso del concetto di economie esterne per spiegare la proliferazione di piccole imprese principalmente all'interno di un settore in località come Prato (tessile) o Cascina (mobili).

Tuttavia, alcune criticità sono ravvisabili in ordine a nuovi processi sociali e del lavoro che sono andati via via modificandosi anche in relazione all'avanzare di dinamiche di impresa orientate a una dimensione non locale e che hanno in parte minato alcuni capisaldi della co-creazione di valore distrettuale. Uno di questi era legato al fatto che negli anni Novanta il migliore stato di benessere della popolazione spostava la tipologia dei consumi da beni standardizzati a beni personalizzati, ad esempio particolari prodotti alla persona e per la casa la cui produzione specializzata era una caratteristica di forza dei distretti legata sia all'alta flessibilità, sia alla disponibilità di una forza lavoro artigianale di eccellenza. Nei recenti anni la trasformazione digitale di molti processi aziendali e il diffondersi di una economia basata sulla conoscenza hanno identificato una nuova classe di lavoratori, i knowledge workers, quali artigiani moderni omologhi di quelli che hanno contribuito allo sviluppo distrettuale. Alcuni fattori geografici distintivi di alcuni territori risultano catalizzatori dei processi di concentrazione di tali lavoratori. Come infatti sostiene Moretti (2013), consolidate teorie sulla agglomerazione, che mettono in evidenza vantaggi derivanti alla concentrazione di imprese in termini di filiere e reti, di produzione omogenea, di clustering, spiegano fenomeni di intensità moderata, come ad esempio la concentrazione dell'impresa dell'entertainment nella città di Los Angeles, ma non giustificano altrettanti fenomeni di intensità elevata, elevatissima che caratterizzano l'economia della innovazione. Emblematica in tal senso risulta essere ad esempio l'industria mondiale delle nanotecnologie concentrata in meno di 10 aree metropolitane. Tra tali fattori distinti- vi rientra sicuramente la percentuale di popolazione ad alta scolarità che rientra tra quelle selezionate nell'indagine svolta descritta al paragrafo successivo.

Il framework distrettuale è dunque molto variegato e alterna punti di forza a criticità da fronteggiare; tuttavia rappresenta una modalità organizzativa della produzione, e ormai anche dei servizi, che è di rilievo rispetto ai volumi produttivi in gioco a livello generale di sistemaPaese.

La profilazione di raggruppamenti omogenei distrettuali aventi come denominatore comune alcuni aspetti, in particolar modo legati alla innovazione, può costituire un valore aggiunto di analisi ed è questo aspetto che è stato indagato.

L'iter metodologico seguito prevede prioritariamente un trattamento analitico dei valori delle variabili distrettuali e successivamente una riflessione geografica sui risultati trovati.

\section{L'analisi sui distretti industriali italiani}

\subsection{Il modello per la valutazione di isomorfismi geografica- mente non prossimi}

Come descritto nella sezione introduttiva, il modello del sistema distrettuale proposto è quello di una rete costituita dai "nodi-distretto" e dai "ramiaffinità tra i distretti”. La scelta relativa alla ricerca di una prossimità non di tipo geografico ma riferita alle variabili distrettuali è dettata da una duplice finalità: da un lato consente di arricchire il dibattito scientifico sull'argomento mediante una profilazione del fenomeno distrettuale a scala nazionale in termini di convergenze e divergenze, e dall'altro mette a disposizione di manager d'impresa e addetti ai lavori operanti nei distretti alcune informazioni utili all'eventuale perseguimento di strategie comuni.

Come sostenuto da Padgett e Powell "nel breve periodo, gli attori creano relazioni; a lungo termine, le relazioni creano l'attore" $(2012,3)$ e l'importanza delle relazioni non risiede ovviamente solo in quelle basate sulla prossimità geografica (Balland et al. 2015).

Pertanto, secondo la prospettiva condotta di sistema-distrettuale configurato come rete, la metodologia di analisi non può che essere quella della network analysis e la metrica individuata dovrà essere il coefficiente di clustering, per una cui descrizione dettagliata si rimanda al lavoro di Watts e Strogatz (1998).

In estrema sintesi, nella teoria dei grafi il coefficiente di clustering esprime una misura del grado in cui i nodi di un grafo tendono ad essere connessi fra 
loro. Esso può essere misurato in due modi diversi: globale e locale. Quello globale descrive in generale l'intensità del fenomeno di clustering nella rete, mentre quello locale riguarda il livello di radicamento dei singoli nodi. Il primo si basa su triple di nodi tra loro connessi da due o da tre collegamenti (rispettivamente tripla aperta o tripla chiusa) e viene valutato come il rapporto tra il numero di triple chiuse e il numero totale di triple (somma di quelle aperte e chiuse). Il primo tentativo di misurarlo fu effettuato da Luce e Perry (1949). Tale metodo può essere applicato sia ai grafi orientati che non orientati, come nel caso distrettuale in cui si è interessati solo a rilevare la presenza o meno di una mutua affinità.

Il coefficiente di clustering locale di un nodo esprime la capacità dei suoi nodi vicini di formare un grafo nel quale ogni vertice risulti essere collegato a tutti i vertici rimanenti.

In altri termini tali metriche sono utili a misurare la capacità di una rete di essere interconnessa e l'influenza di ciascun nodo nella generazione di collegamenti tra nodi ad esso prossimi.

La declinazione di tale metodologia al caso in esame è stata fatta trasponendo al concetto di relazione quello di affinità e, pertanto, il criterio abilitante l'individuazione di un isomorfismo, come detto, è l'omogeneità di almeno una (one at least) variabile tra le 4 che verranno prese in esame nell'analisi. L'evidenza suggerisce che nella maggior parte delle reti del mondo reale i nodi tendono a creare gruppi fortemente convergenti e caratterizzati da una densità di affinità (i collegamenti della rete) relativamente alta: e dunque il coefficiente di clustering delle reti reali tende ad essere maggiore rispetto a quello dei raggruppamenti in cui le affinità sono generate casualmente (Holland, Leinhardt 1971; Watts, Strogatz, 1998).

In letteratura scientifica diversi studi analizzano le dinamiche e i modelli di valutazione della capacità di raggrupparsi in cluster (si vedano i recenti lavori di Louch 2000; Snijders 2001; Snijders et al. 2006; analisi più datate sono riscontrabili in Heider 1946; Holland, Leinhardt 1970; Feld 1981).

Un grafo $G=(V, E)$ \} consiste formalmente di un insieme $\mathrm{V}$ di vertici e un insieme $\mathrm{E}$ di collegamenti. Un collegamento $e_{i j}$ connette un vertice $v_{i}$ con un vertice $v_{j}$

L'insieme $\mathrm{N}_{\mathrm{i}}$ dei vicini di un vertice vi è definito come l'insieme dei nodi direttamente connessi ad esso: $\mathrm{N}_{\mathrm{i}}=\left\{\mathrm{v}_{\mathrm{j}}: \mathrm{e}_{\mathrm{ij}} \in \mathrm{E}, \mathrm{e}_{\mathrm{ji}} \in \mathrm{E}\right\}$.

Definita $k_{i}$ come la cardinalità di $\left|N_{i}\right|$, ovvero il numero di vicini di un vertice $\mathrm{v}_{\mathrm{i}}$, il coefficiente di clustering locale $\mathrm{Cl}_{\mathrm{i}}$ di un vertice $\mathrm{v}_{\mathrm{i}}$ è dato dal numero di collegamenti fra i membri di $\mathrm{N}_{\mathrm{i}}$ fratto il numero di colle-

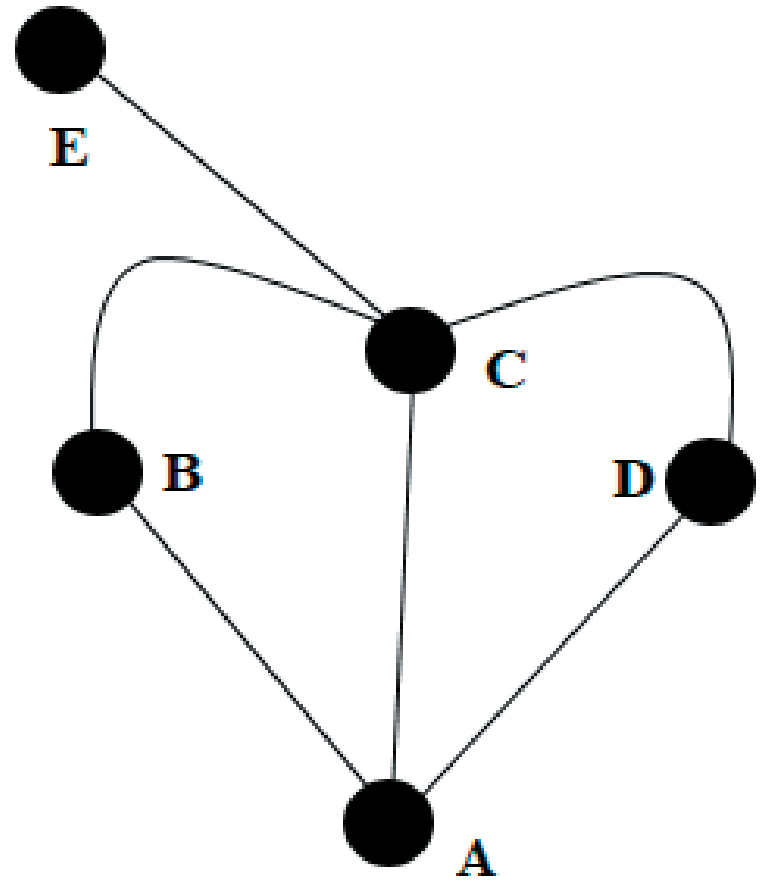

Figura 1. Esempio calcolo del coefficiente di clustering. Fonte: elaborazione dell'autore

gamenti potenziali fra loro.

In un grafo orientato $\mathrm{e}_{\mathrm{ij}}$ è distinto da $\mathrm{e}_{\mathrm{ij}}$, dunque per ogni $\mathrm{N}_{\mathrm{i}}$ restano definiti $\mathrm{k}_{\mathrm{i}}\left(\mathrm{k}_{\mathrm{i}}-1\right)$ collegamenti possibili fra i suoi membri. Di conseguenza, il coefficiente di clustering locale per grafi orientati è dato da:

$$
C l_{i}=\frac{\left|\left\{e_{j k}: v_{j}, v_{k}, \in N_{i}, e_{j k} \in E\right\}\right|}{(K i) \cdot(K i-1)}
$$

La proprietà caratteristica di un grafo non orientato è invece che $\mathrm{e}_{\mathrm{ij}}$ e $\mathrm{e}_{\mathrm{ji}}$ sono considerati identici, dunque per ogni $\mathrm{N}_{\mathrm{i}}$ restano individuati $\mathrm{k}_{\mathrm{i}}\left(\mathrm{k}_{\mathrm{i}}-1\right) / 2$ collegamenti possibili fra i suoi membri. Di conseguenza, il coefficiente di clustering locale per grafi non orientati è dato da:

$$
C l_{i}=\frac{2\left|\left\{e_{j k}: v_{j}, v_{k}, \in N_{i}, e_{j k} \in E\right\}\right|}{(K i) \cdot(K i-1)}
$$

A titolo di esempio la figura 1 rappresenta uno schema di rete in cui il nodo A possiede 3 collegamenti (con i nodi $\mathrm{B}, \mathrm{C}$ e D). La capacità del nodo A di generare collegamenti tra i suoi nodi vicini è concretizzata, in questo caso esemplificativo, dai 2 collegamenti $\mathrm{BC}$ e CD, e per- 
tanto i coefficienti di clustering riferiti ai vari nodi assumo i seguenti valori:

$$
C l_{A}=2 \frac{2}{3 \cdot(3-1)}=2 / 3=0,66 ; \mathrm{Cl}^{\mathrm{B}}=1 ; \mathrm{Cl}^{\mathrm{E}}=0 ; \mathrm{Cl}=2 / 6=0,33 \text { e } \mathrm{Cl}^{\mathrm{D}}=1 \text {. }
$$

Pertanto, il campo di variazione dei valori del coefficiente oscilla tra 0 (il numeratore è nullo) - condizione nella quale il nodo sul quale è calcolato il coefficiente (clustering locale) non induce alcuna affinità (legame) tra i nodi ai quali è esso affine - ed 1 (numeratore e denominatore si eguagliano), condizione nella quale invece esso induce ogni possibile convergenza compatibile con l'architettura degli altri nodi in gioco.

Sono presentate due misure alternative calcolate attraverso il software Ucinet ${ }^{\oplus}$, l'Overall graph clustering coefficient che rappresenta la media dei valori dei coefficienti riferiti a tutti nodi del cluster, in altre parole come direbbero gli statistici "la media fuori tutto", e la versione "ponderata", nota come Weighted Overall graph clustering coefficient che attribuisce un peso in relazione al numero di triple di nodi nel cluster nel quale i nodi vengono ad essere centrali (per i dettagli si veda Watts, Strogatz 1998) ${ }^{4}$.

Indipendentemente da dettagli analitici il cui approfondimento esula dalla loro funzione prettamente strumentale qui necessaria, il significato geografico atteso in relazione alla valutazione di tali metriche è che quanto più queste saranno elevate tanto più il raggruppamento distrettuale sarà omogeneo, ossia i distretti ad esso appartenenti saranno affini. Tale grado di affinità andrà poi confrontato con la loro relativa ubicazione geografica per analizzarne una eventuale dipendenza o indipendenza dal contesto territoriale (in termini di ricchezza del territorio e di dinamismo imprenditoriale) e settoriale (i distretti sono classificati per settori, agroalimentare, ICT, meccanica, sistema moda e sistema casa).

\subsection{Analisi svolta}

L'analisi svolta è consistita in una serie di passaggi metodologici sequenziali brevemente descritti di seguito, mentre le considerazioni riflessive di tipo geografico sono riportate nel paragrafo successivo.

Il punto di partenza è costituito dalla individuazione delle variabili di contesto distrettuale necessarie alla individuazione degli isomorfismi ricercati.

\footnotetext{
${ }^{4}$ Questa seconda metrica vuole rappresentare una mera verifica ulteriore rispetto alla già soddisfacente prima metrica finalizzata a verificare l'assenza di eventuali anomalie che verrebbero mascherate dalla media fuori tutto dei valori.
}

Nella sezione 8 del Rapporto fonte dei dati viene analizzata la propensione all'innovazione dei contesti territoriali in cui operano le imprese distrettuali focalizzando l'analisi effettuata nelle precedenti edizioni dell'Osservatorio e sintetizzata dall'Indice della Qualità della Vita delle imprese nei Distretti. Sono definite e rilevate 16 variabili, di cui alcune a scala provinciale e altre a scala regionale caratterizzanti i vari distretti censiti classificati per settore (A: Agroalimentare; ICT: Tecnologie digitali; MEC: Metalmeccanica; SC: Sistema Casa; SM: Sistema Moda).

Per ridurre il calcolo computazionale senza perdere in significatività della base dati si sono scelte 4 variabili tra le 16 censite dal Rapporto e sono: la Quota \% di Imprese High-Tech (definita in tale contributo ai fini dell'analisi come QIHT), il tasso \% di Sviluppo di Imprese High-Tech (SIHT), la \% di Popolazione ad Alta Scolarità (PAS), la percentuale di Spesa in $R \& S$ $(\mathrm{SRS})^{5}$.

La selezione è stata operata in due passi successivi di elaborazione della base dati fornita dal Rapporto costituita dalla matrice avente per colonne le 16 variabili totali e per righe tutti i distretti italiani censiti. Un primo sottoinsieme di variabili è stato ottenuto attraverso una routine software in due step, atta in prima istanza a sviluppare l'intero piano combinatoriale dei possibili confronti tra i valori delle variabili distrettuali e successivamente a selezionare quelle relative agli esiti più prossimi risultanti dalla elaborazione.

In secondo luogo, si è operata una ulteriore riduzione ad un nucleo rappresentativo della particolare prospettiva di indagine data alla ricerca inerente aspetti legati alla innovazione dei distretti. La quota di imprese innovative, il tipo di background culturale della popolazione e la spesa in ricerca e sviluppo rappresentano, infatti, variabili di stock che ben fotografano uno stadio di innovazione d'impresa consolidato in regime statico, mentre il tasso di sviluppo rappresenta una variabile di flusso e fornisce, pertanto, indicazioni relative alla derivata del fenomeno, ossia attiene al rapporto tra intensità di sviluppo e unità di tempo nel quale esso avviene, rivelandosi così una buona proxy di assenza o presenza di dinamismo imprenditoriale nell'area distrettuale.

\footnotetext{
${ }^{5}$ Quota imprese high-tech (\%): percentuale di imprese high-tech su totale imprese. Scala: provinciale. Tasso di sviluppo di imprese high-tech (\%): numero di imprese Iscritte nel 2011 meno imprese cessate non d'ufficio. Scala: provinciale. Popolazione ad alta scolarità (\%): Diploma di scuola superiore, titolo universitario accademico e titolo superiore. Scala: provinciale. Spesa R\&S (\%): Spesa per ricerca e sviluppo intra-muros in \% Pil a prezzi correnti. Scala: regionale.
} 


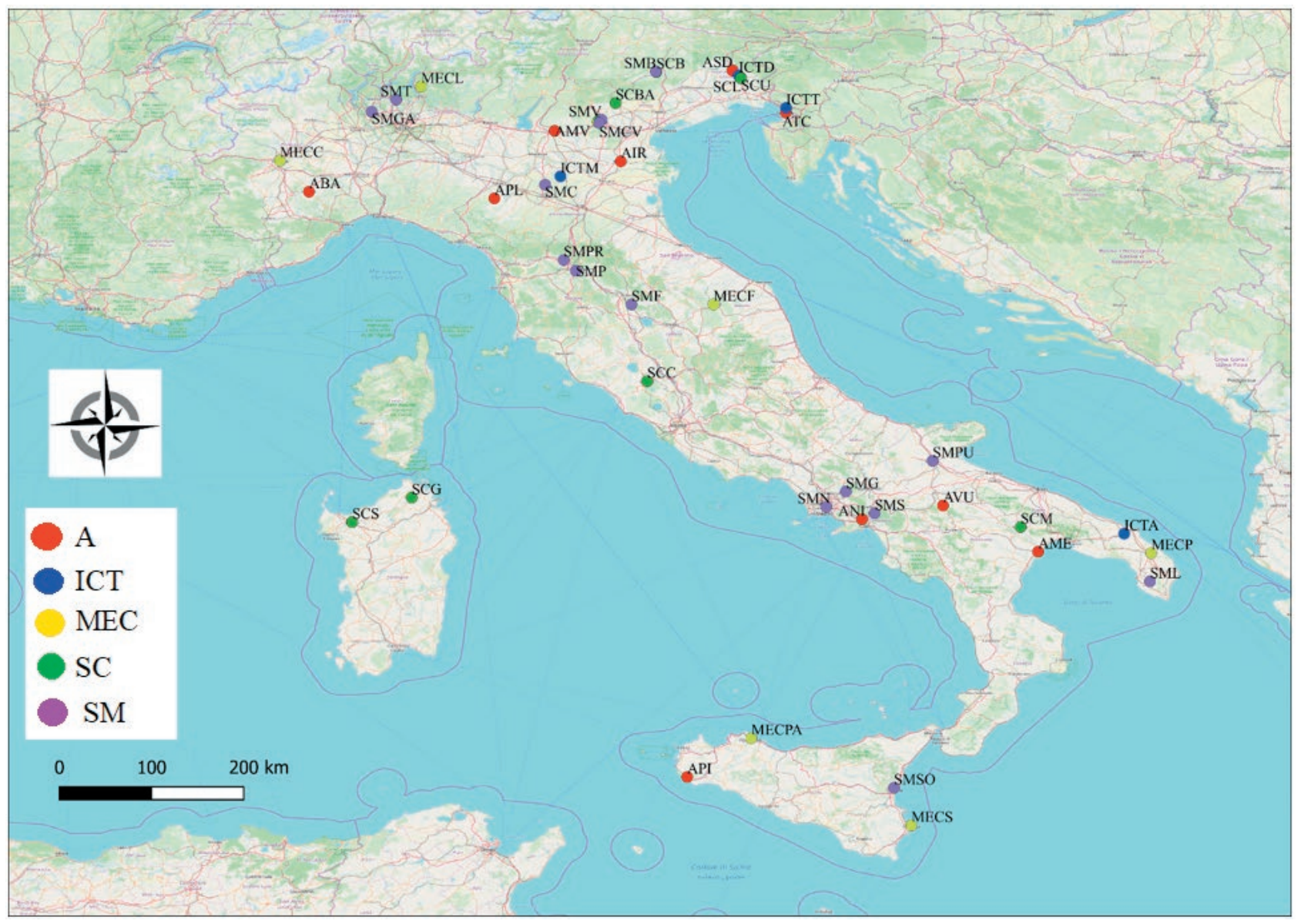

Figura 2. Distribuzione geografica dei distretti italiani. Fonte: elaborazione dellautore su dati Unioncamere.

Il risultato di questa prima selezione ha portato alla matrice dati di partenza distretti-variabili, riportata in appendice 1, la cui distribuzione geografica è riportata nel cartogramma di figura 2 , dal quale risulta una distribuzione distrettuale estesa su tutta la Penisola, che presenta cluster settoriali in funzione dell'area geografica di riferimento.

Gli interrogativi di ricerca posti a base dell'analisi sono stati sintetizzati nella formulazione (2) nella quale sono passate a verifica tre condizioni. La condizione di partenza, $\left(\mathrm{H}_{\text {iniz. }}\right)$, è ovviamente la verifica della esistenza di isomorfismi geograficamente non prossimi e questa è già dimostrata, in forma grossolana, dal primo passo di selezione delle variabili distrettuali opportune. Restano da verificare le altre due ipotesi di ricerca $\left(\mathrm{H}_{1}\right.$ e $\left.\mathrm{H}_{2}\right)$ che di fatto rappresentano una formulazione analitico-geografica ad alta sensibilità rispetto alla grossolana ipotesi iniziale.

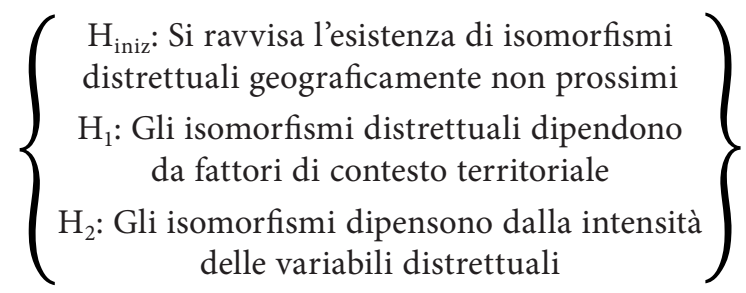

A partire dal set di valori della tabella in appendice 1 si è applicato un metodo di suddivisione in classi. La scelta del loro numero è stata guidata dalla necessità di soddisfare due esigenze antitetiche: da un lato quella di ridurre la complessità computazionale scaturente da un numero elevato di classi, e dall'altro dalla volontà di rilevare una eventuale variazione del fenomeno contemplando l'ipotesi di scenario non solo lineare (intercettabile con due sole classi). 
Tabella 1. Coefficienti di clustering. Fonte: elaborazione dell'autore con software Ucinet ${ }^{\circ}$.

\begin{tabular}{|c|c|c|c|}
\hline Variabile & Livello isomorfismo & $\begin{array}{c}\text { Overall graph } \\
\text { clustering coefficient }\end{array}$ & $\begin{array}{l}\text { Weighted Overall graph } \\
\text { clustering coefficient }\end{array}$ \\
\hline \multirow{3}{*}{ QIHT } & B & 0.502 & 0.474 \\
\hline & M & 0.363 & 0.358 \\
\hline & A & $(0.512)$ & $(0.512)$ \\
\hline \multirow{3}{*}{ SIHT } & B & 0.515 & 0.515 \\
\hline & M & 0.462 & 0.451 \\
\hline & A & $(0.697)$ & $(0.613)$ \\
\hline \multirow{3}{*}{ PAS } & B & $(0.526)$ & $(0.526)$ \\
\hline & M & 0.506 & 0.505 \\
\hline & A & 0.511 & 0.509 \\
\hline \multirow{3}{*}{ SRS } & B & 0.561 & 0.561 \\
\hline & M & $(0.572)$ & $(0.572)$ \\
\hline & $\mathrm{A}$ & 0.536 & 0.534 \\
\hline
\end{tabular}

La scelta è, pertanto, ricaduta sul numero tre ${ }^{6}$ distinguendo fascia bassa (B), media (M) e alta (A) per ciascuno dei 4 scenari QIHT SIHT PAS SRS, per i quali sono state calcolate le metriche di clustering descritte al paragrafo precedente (Tab. 1) e successivamente confrontate con le relative distribuzioni geografiche.

Dall'osservazione dei dati della tabella 1 gli isomorfismi che risultano attenere ad aree di forza sono relativi a un cluster di distretti con elevati valori della variabile Quota di Imprese High Tech e a un cluster di distretti con elevati valori della variabile Sviluppo Imprese High Tech. Mentre quelli che caratterizzano le aree di debolezza riguardano il cluster ottenuto dalla stratificazione per la variabile Popolazione ad Alta scolarità e quello generato stratificando per la variabile Spesa in R\&S.

Al fine di aumentare la risoluzione dell'indagine si sono distinti gli isomorfismi generici, relativi alla tabella 1 , in ulteriori classificazioni atte a differenziare gli isomorfismi perfetti relativi a massima convergenza tra $i$ valori delle diverse variabili all'interno delle 3 fasce di afferenza. Tale condizione è ottenuta per eguaglianza piena dei valori oppure per difetto o eccesso di un errore minimo. Come funzione di errore si è scelta la formula (3) in cui x è il generico valore della variabile e $\mathrm{k}$ la cifra di scostamento ammessa a ritenere quasi uguali le coppie di valori delle variabili confrontate.

${ }^{6}$ Per 3 punti resta, infatti, definita una circonferenza e dunque si evita un eventuale confounding tra variazione lineare e variazione polinomiale.

$$
\mathrm{FE}(\mathrm{x})=\frac{x-(x-k)}{x} 100
$$

In considerazione del fatto che la curva di errore della (2) (in Appendice 2) presenta elevati errori a inizio scala e bassi errori a fondo scala, sono stati considerati ai fini della definizione di isomorfismi perfetti gli errori rispettivamente del $10 \%$ e del $3 \%{ }^{7}$.

Per i cluster relativi a scenari consolidati, quindi o di fascia alta o bassa, sono riportati i diagrammi di clustering nei quali sono stati evidenziati gli isomorfismi perfetti, graficamente rappresentati da rami di spessore maggiore e dai nodi distrettuali interessati anch'essi di dimensione maggiore (Figg. da 3a a 6a per lo scenario di alta fascia e da 7a a 10a per quello di bassa fascia di valori). Al fine di valutare la distribuzione geografica dei vari distretti di ogni cluster nelle due condizioni valoriali delle 4 variabili sono riportati anche i rispettivi cartogrammi corrispondenti (Figg. da $3 \mathrm{~b}$ a $6 \mathrm{~b}$ per lo scenario di fascia alta e da 7 b a 10b per quello di fascia bassa di valori).

\footnotetext{
${ }^{7}$ Ad esempio per $\mathrm{k}=2$ i valori 88 e 86 sono considerati uguali in quanto $(88-(88-2) / 88)^{*} 100$ genera un errore del $2,3 \%$ così come i valori 20 e 18 sono considerati assimilabili in quanto $((20-(20-2)) / 20) * 100$ genera
} un errore del $10 \%$. 


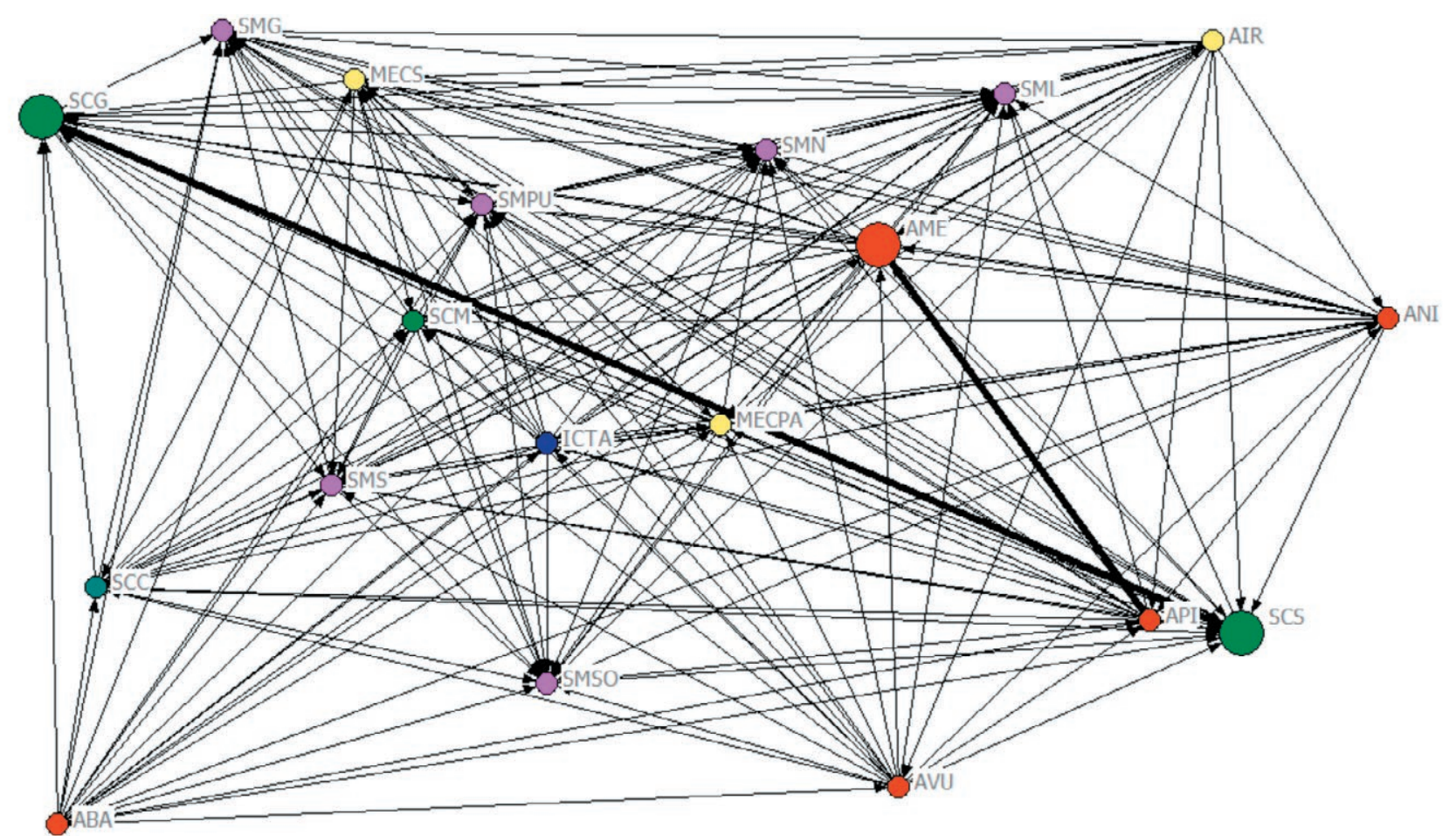

Figura 3a. Diagramma di clustering QIHT-A con evidenza isomorfismi perfetti. Fonte: elaborazione dellautore su dati Unioncamere con software Ucinet ${ }^{\oplus}$.

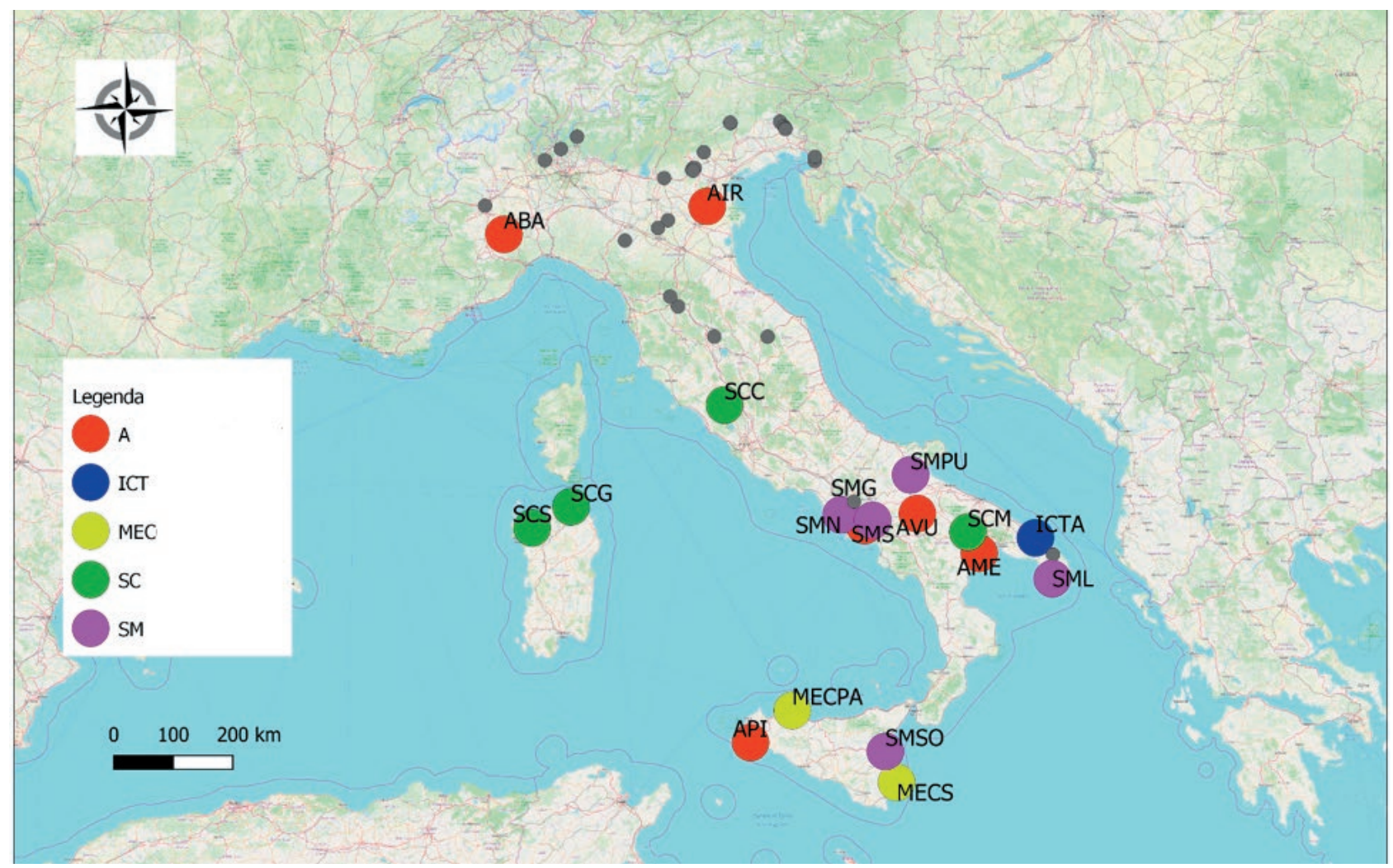

Figura 3b. Distribuzione geografia dei distretti del cluster QIHT-A. Fonte: elaborazione dell'autore su dati Unioncamere. 


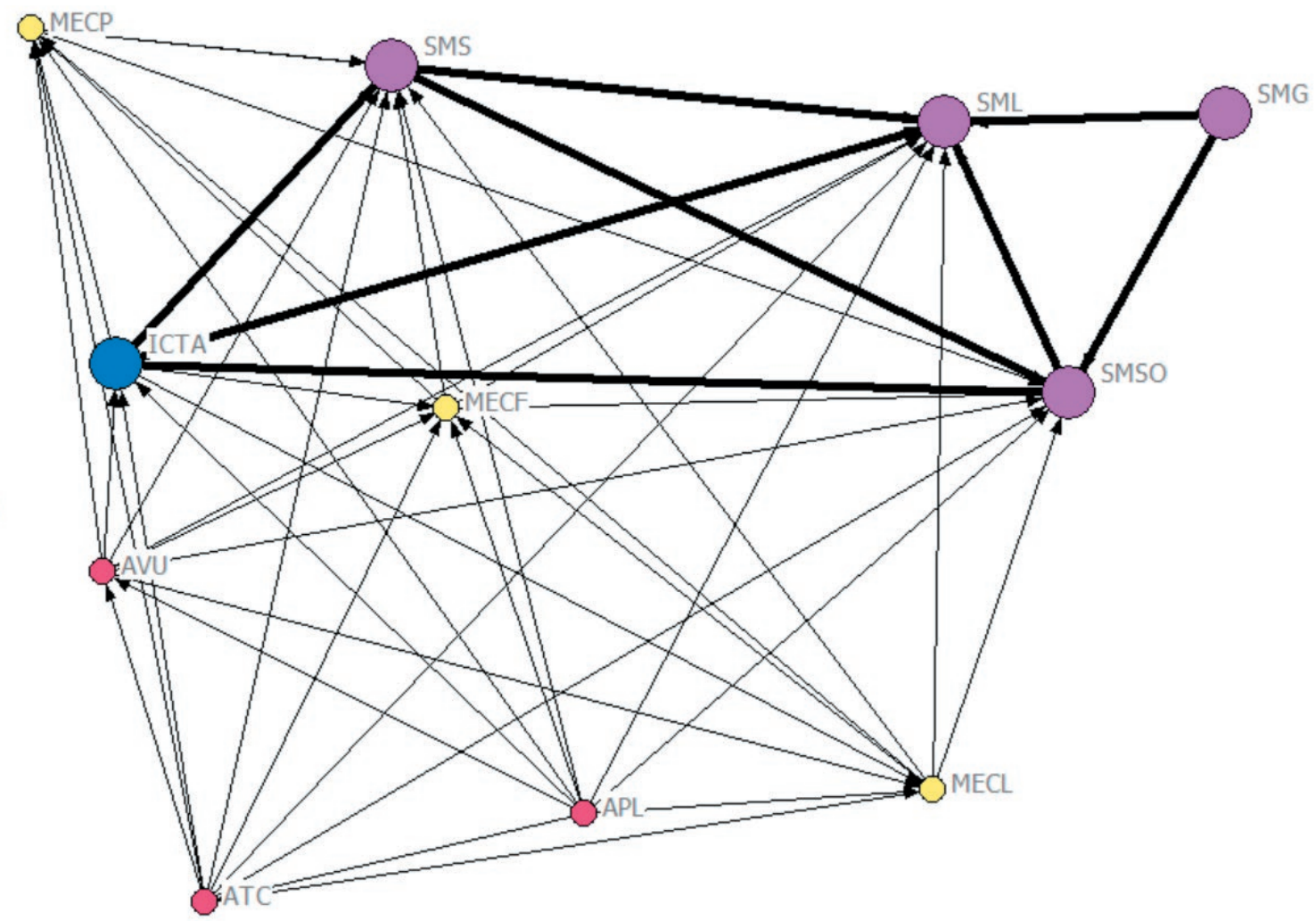

Figura 4a. Diagramma di clustering SIHT-A con evidenza isomorfismi perfetti. Fonte: elaborazione dell'autore su dati Unioncamere con software Ucinet ${ }^{\oplus}$.

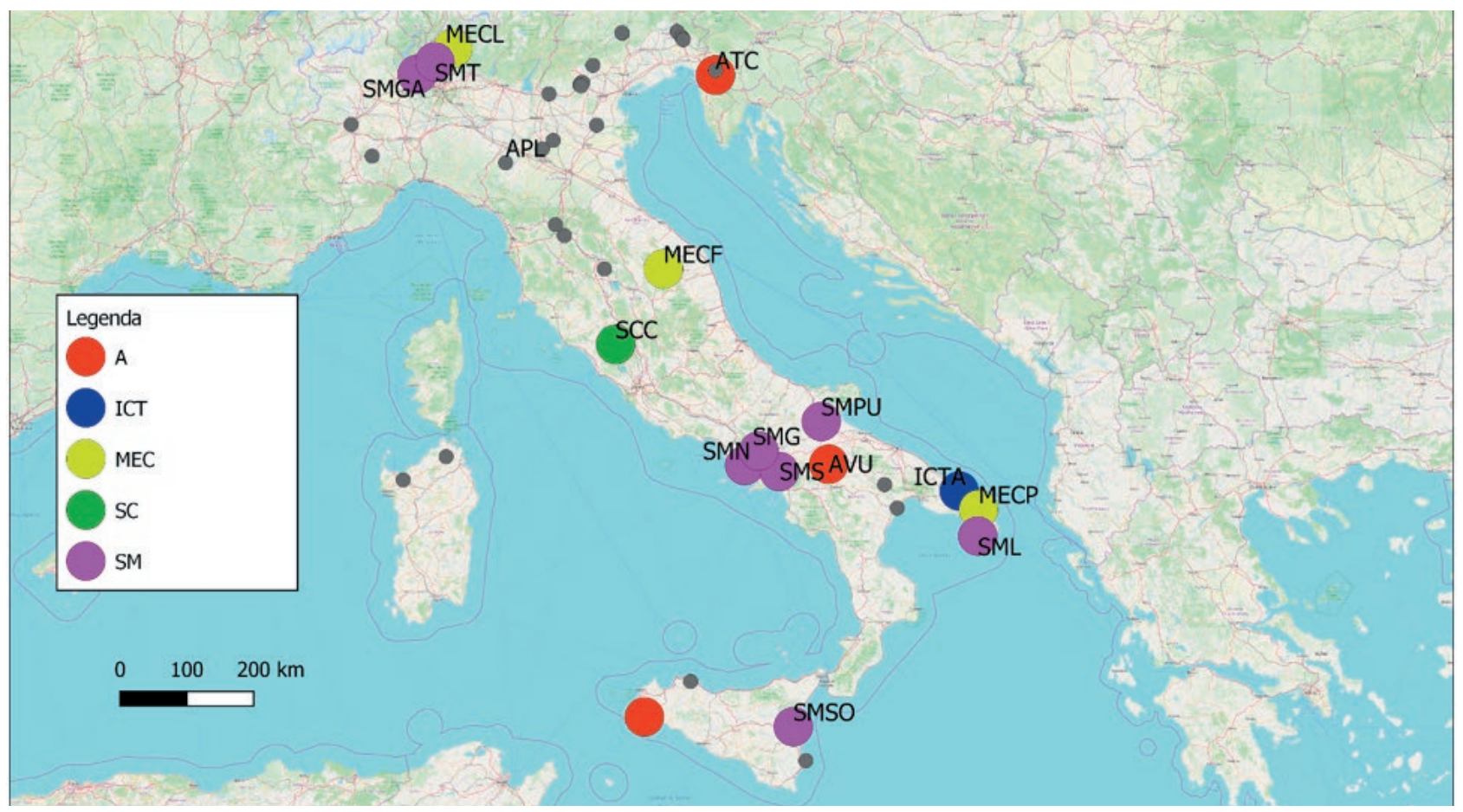

Figura 4b. Distribuzione geografia dei distretti del cluster SIHT-A. Fonte: elaborazione dell’autore su dati Unioncamere. 


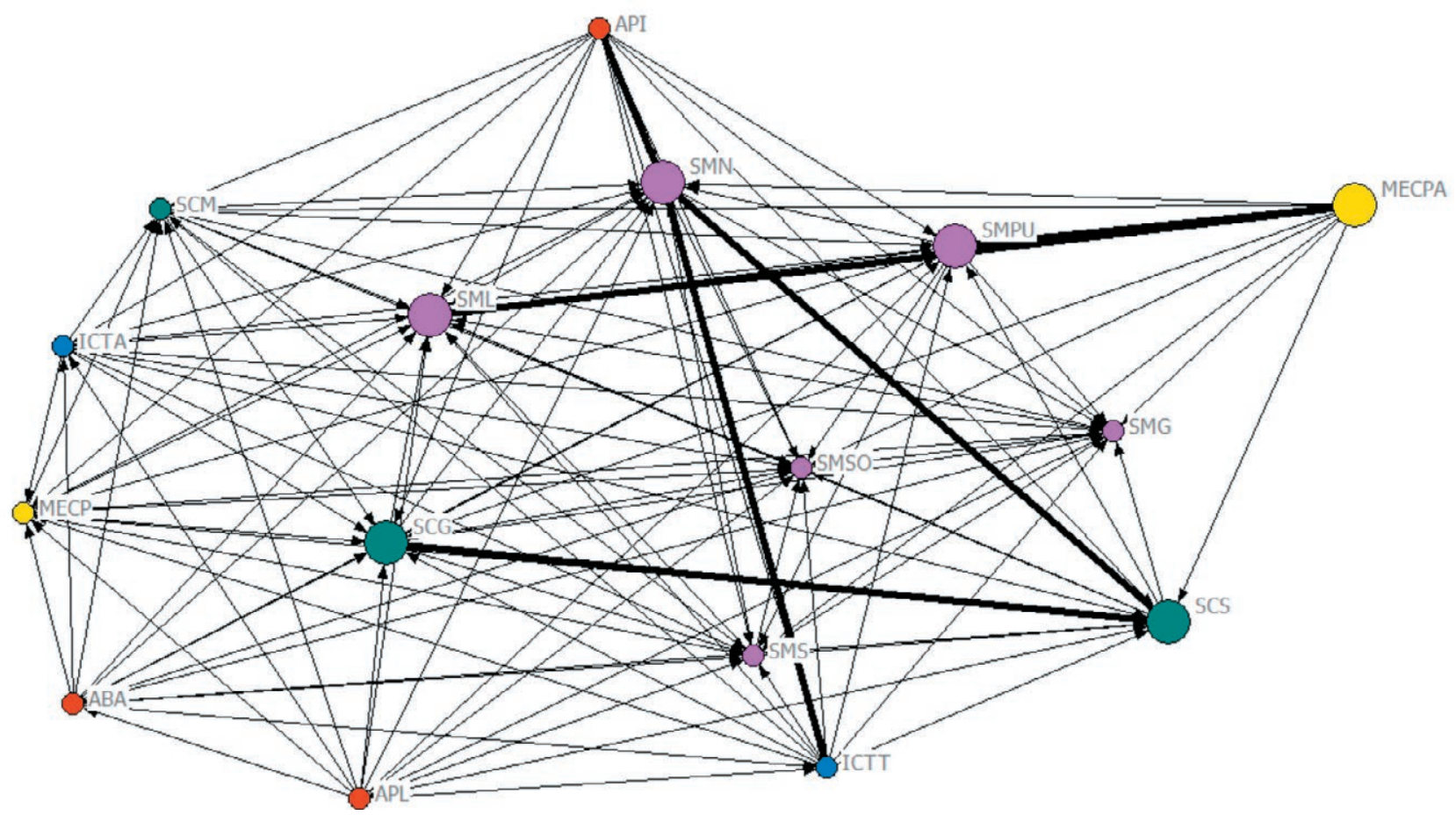

Figura 5a. Diagramma di clustering PAS-A con evidenza isomorfismi perfetti. Fonte: elaborazione dell'autore su dati Unioncamere con software Ucinet ${ }^{\circledR}$.

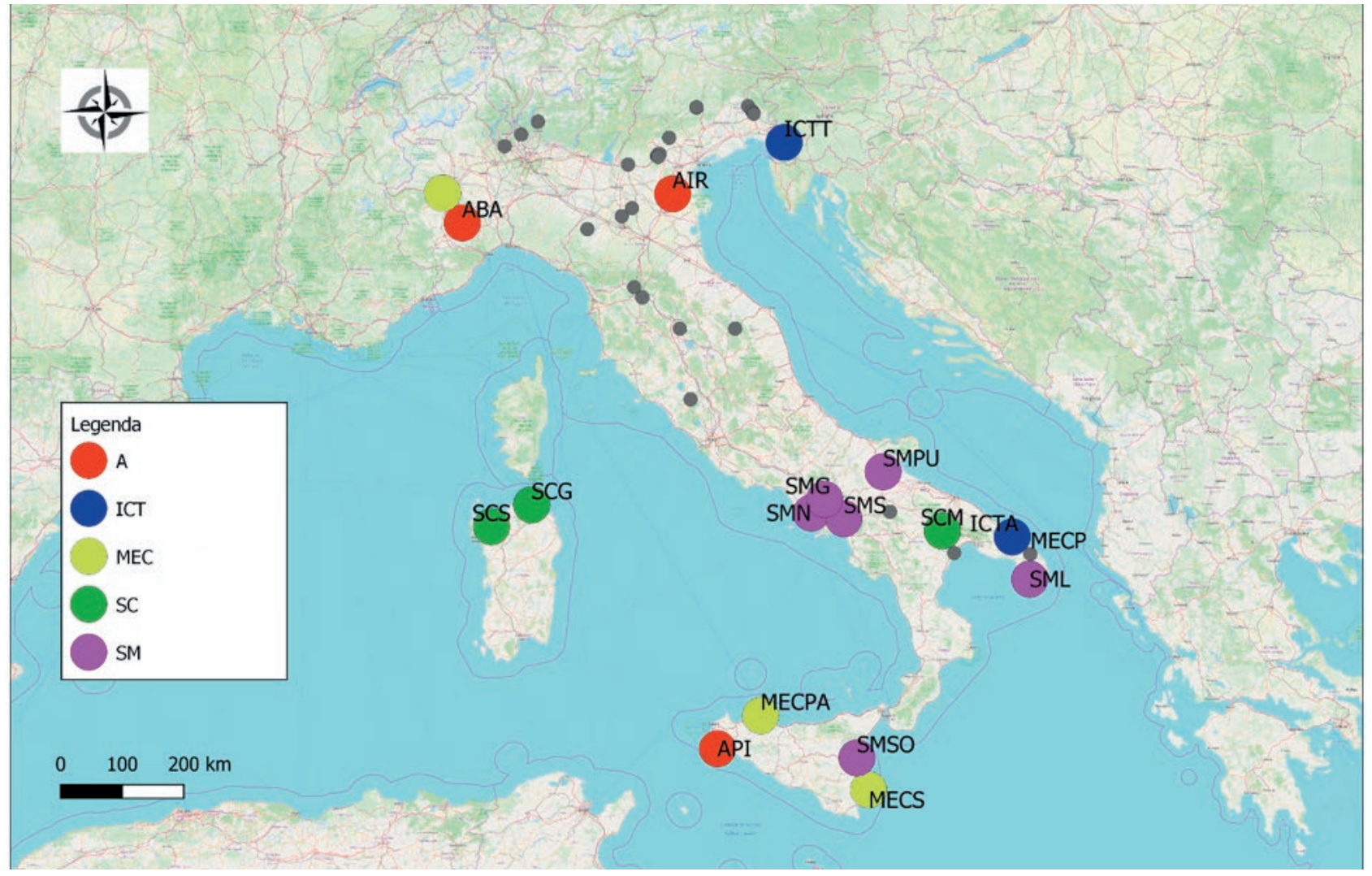

Figura 5b. Distribuzione geografia dei distretti del cluster PAS-A. Fonte: elaborazione dell'autore su dati Unioncamere. 


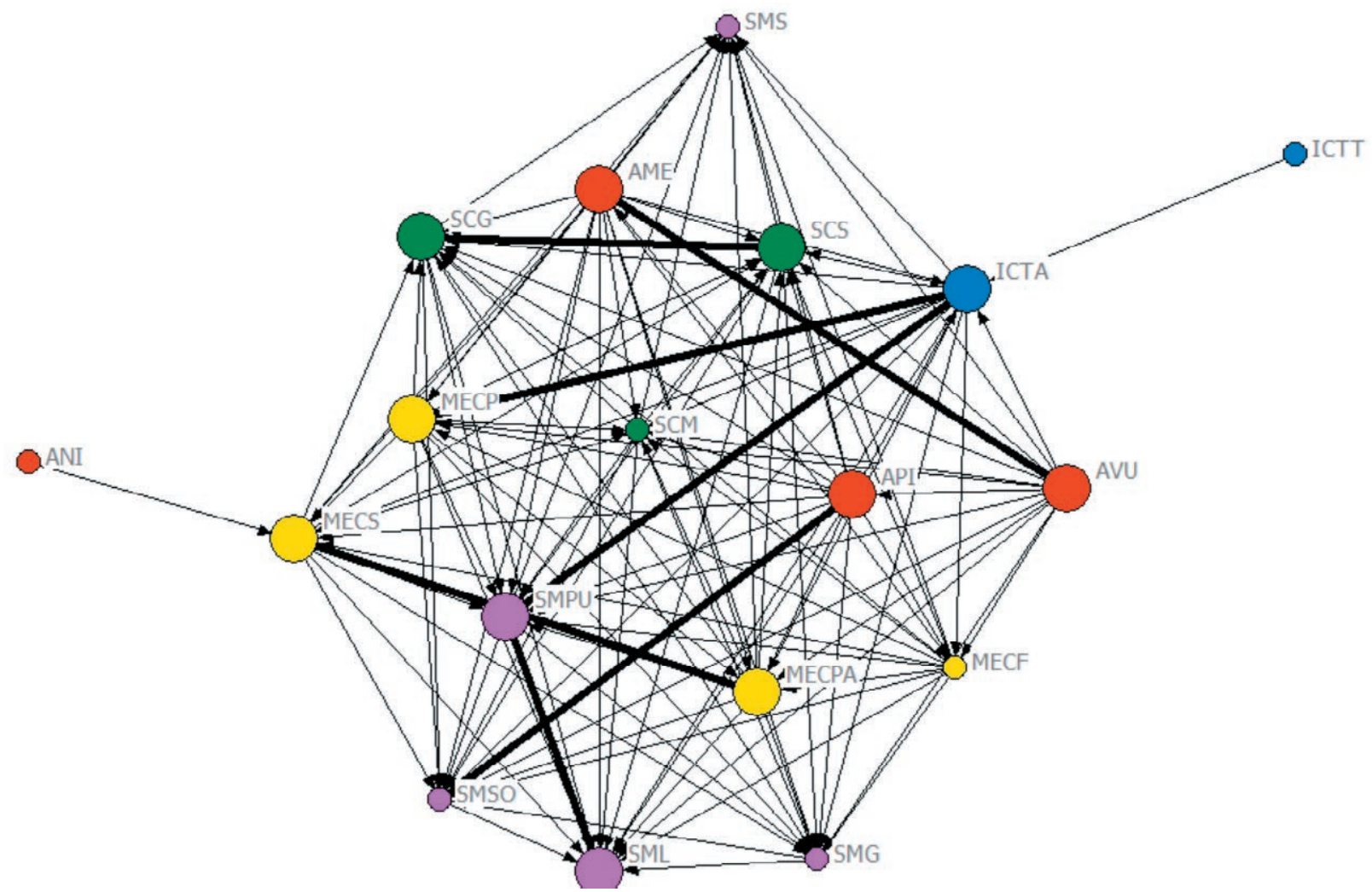

Figura 6a. Diagramma di clustering SRS-A con evidenza isomorfismi perfetti. Fonte: elaborazione dell'autore su dati Unioncamere con software Ucinet ${ }^{\oplus}$.

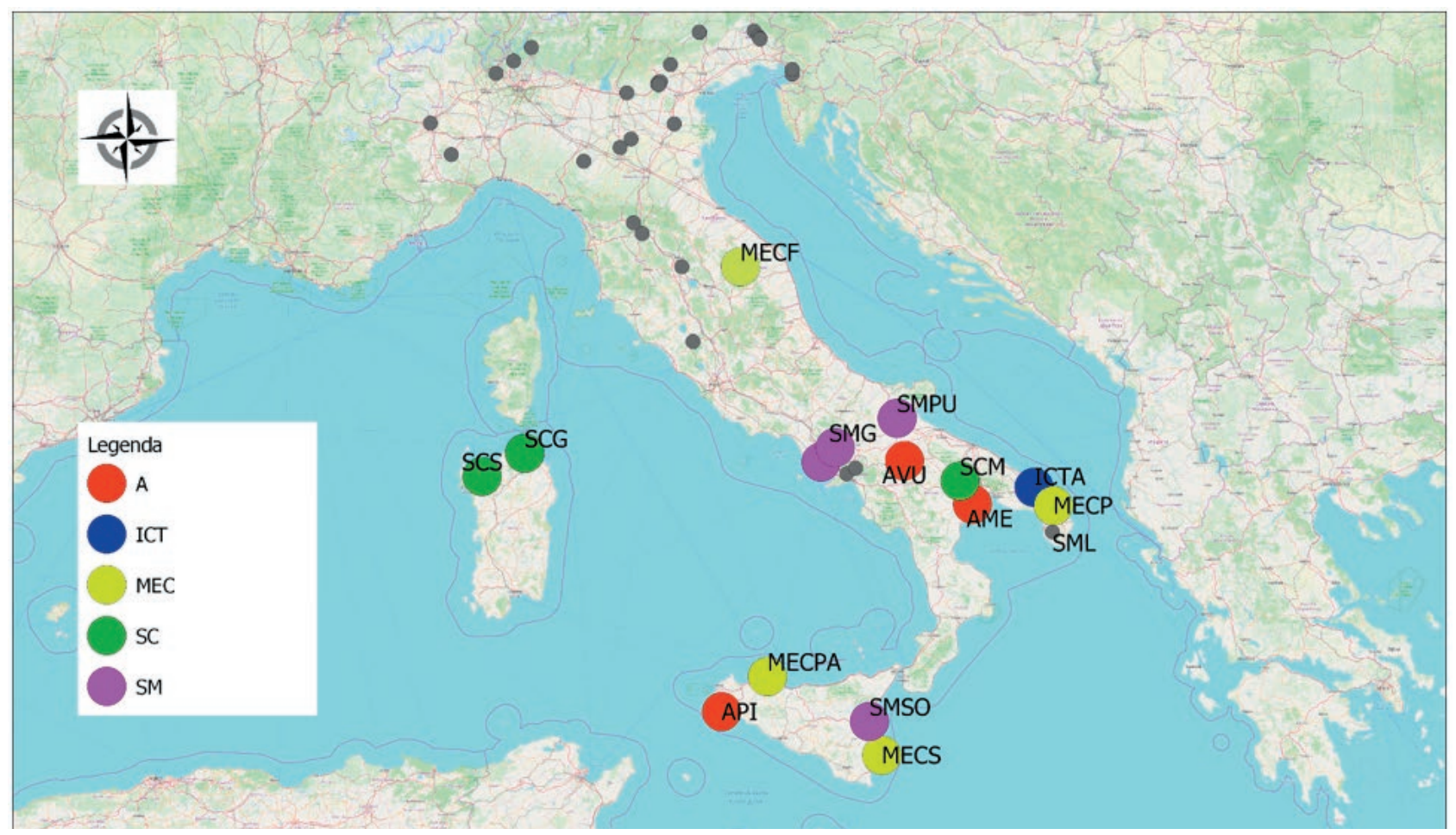

Figura 6b. Distribuzione geografia dei distretti del cluster SRS-A. Fonte: elaborazione dellautore su dati Unioncamere. 


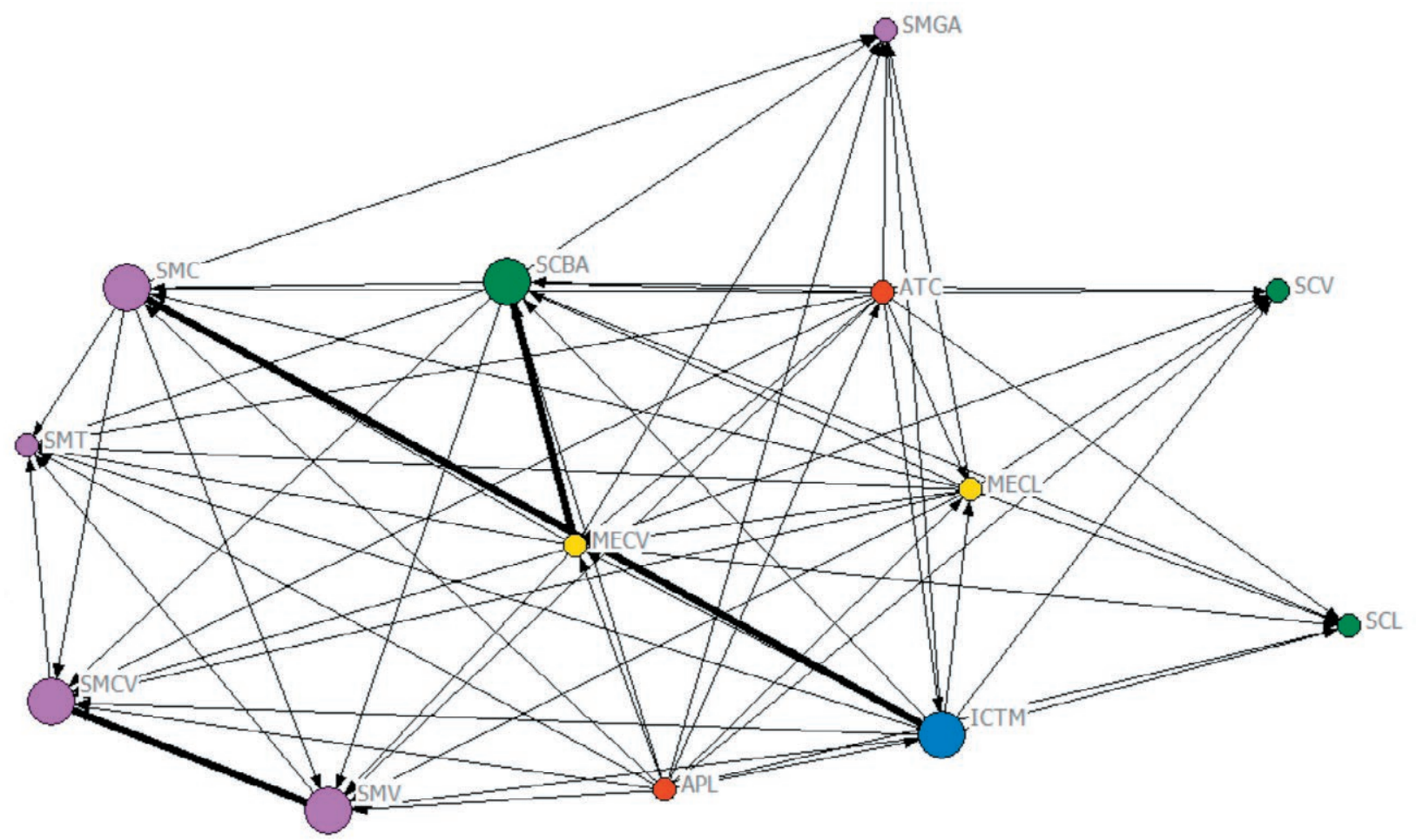

Figura 7a. Diagramma di clustering QIHT-B con evidenza isomorfismi perfetti. Fonte: elaborazione dell'autore su dati Unioncamere con software Ucinet ${ }^{\oplus}$.

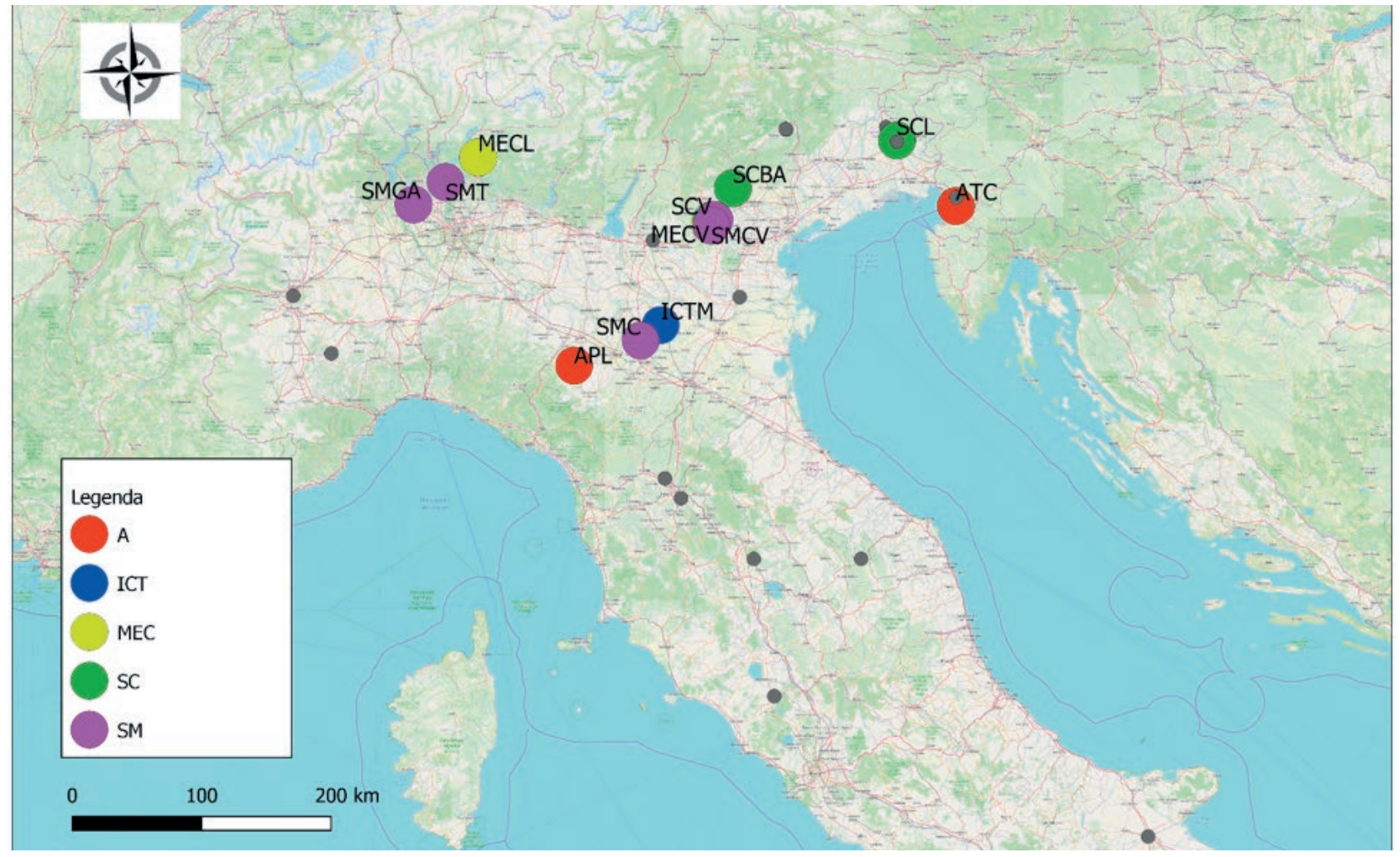

Figura 7b. Distribuzione geografia dei distretti del cluster QIHT -B. Fonte: elaborazione dell'autore su dati Unioncamere. 


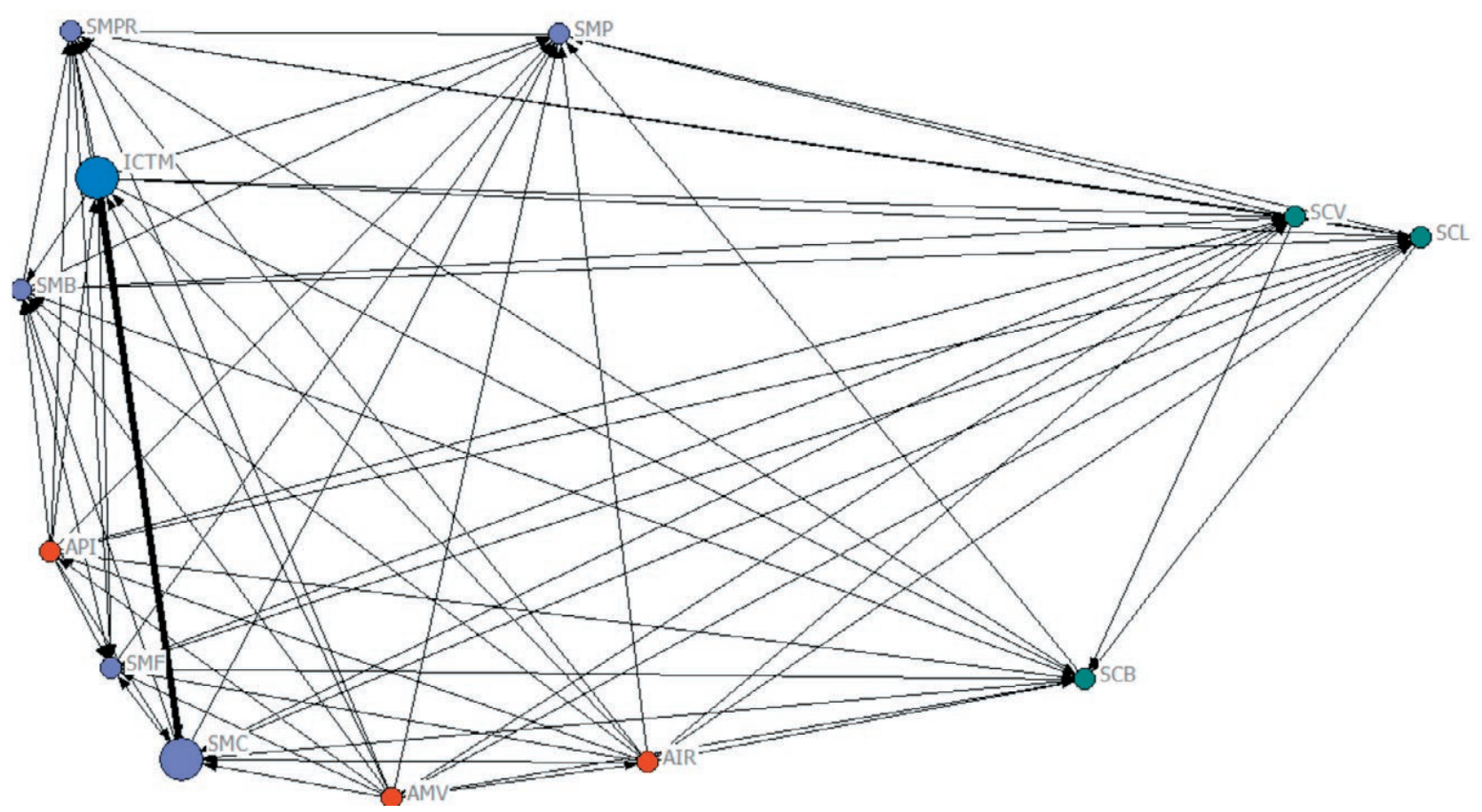

Figura 8a. Diagramma di clustering SIHT-B con evidenza isomorfismi perfetti. Fonte: elaborazione dellautore su dati Unioncamere con software Ucinet ${ }^{\oplus}$.

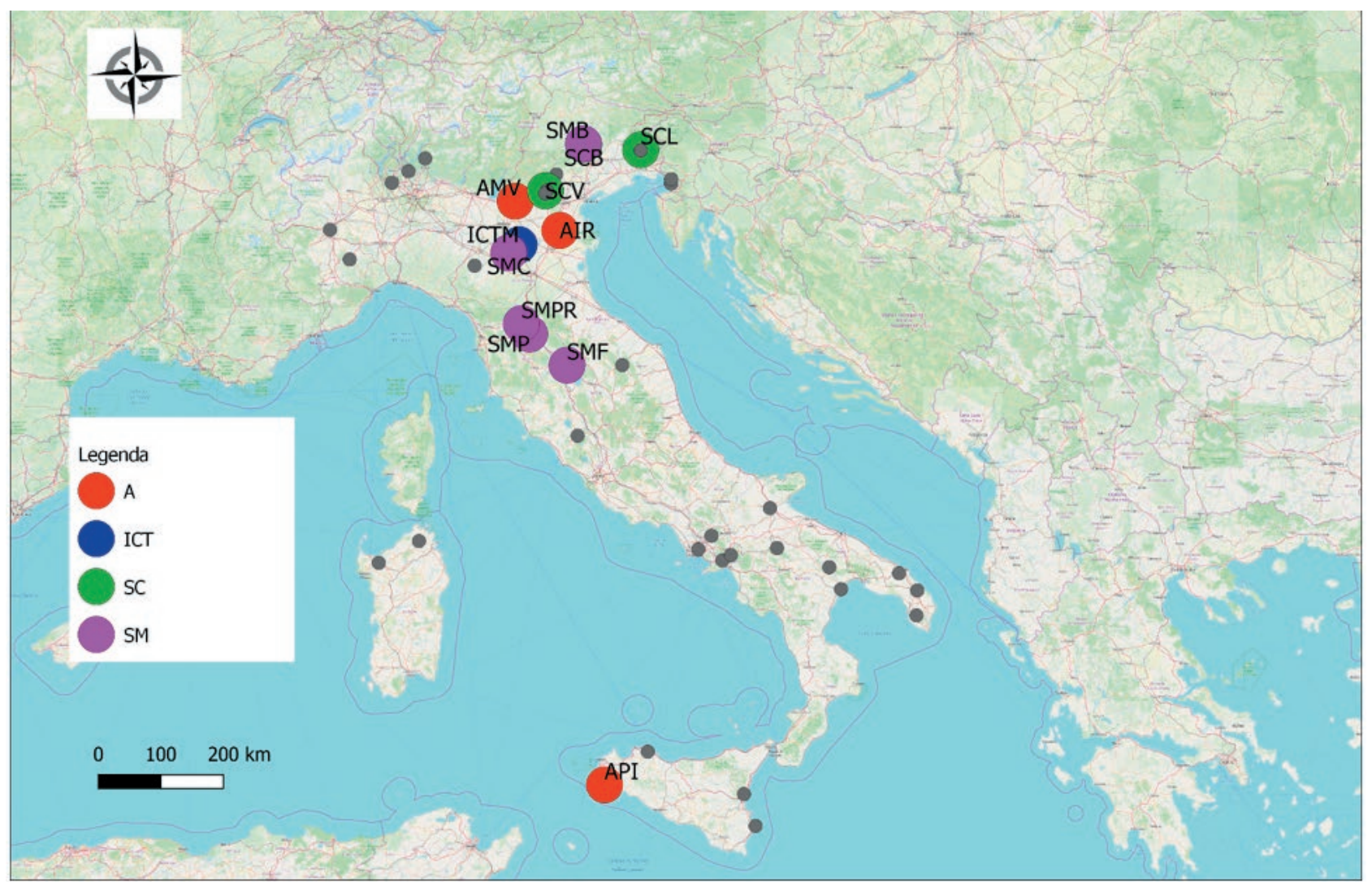

Figura 8b. Distribuzione geografia dei distretti del cluster SIHT-B. Fonte: elaborazione dell'autore su dati Unioncamere. 


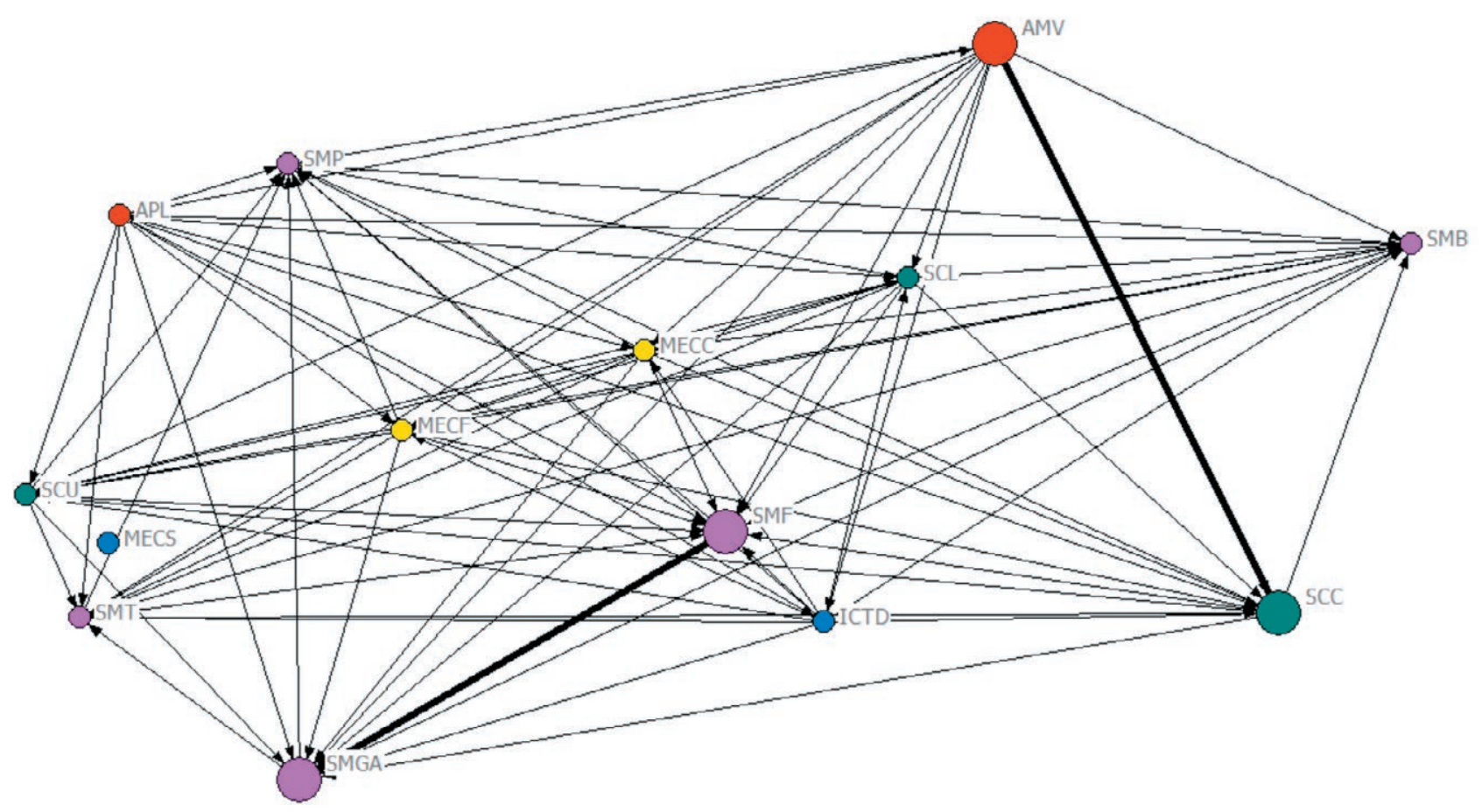

Figura 9a. Diagramma di clustering PAS-B con evidenza isomorfismi perfetti. Fonte: elaborazione dell'autore su dati Unioncamere con software Ucinet ${ }^{\oplus}$.

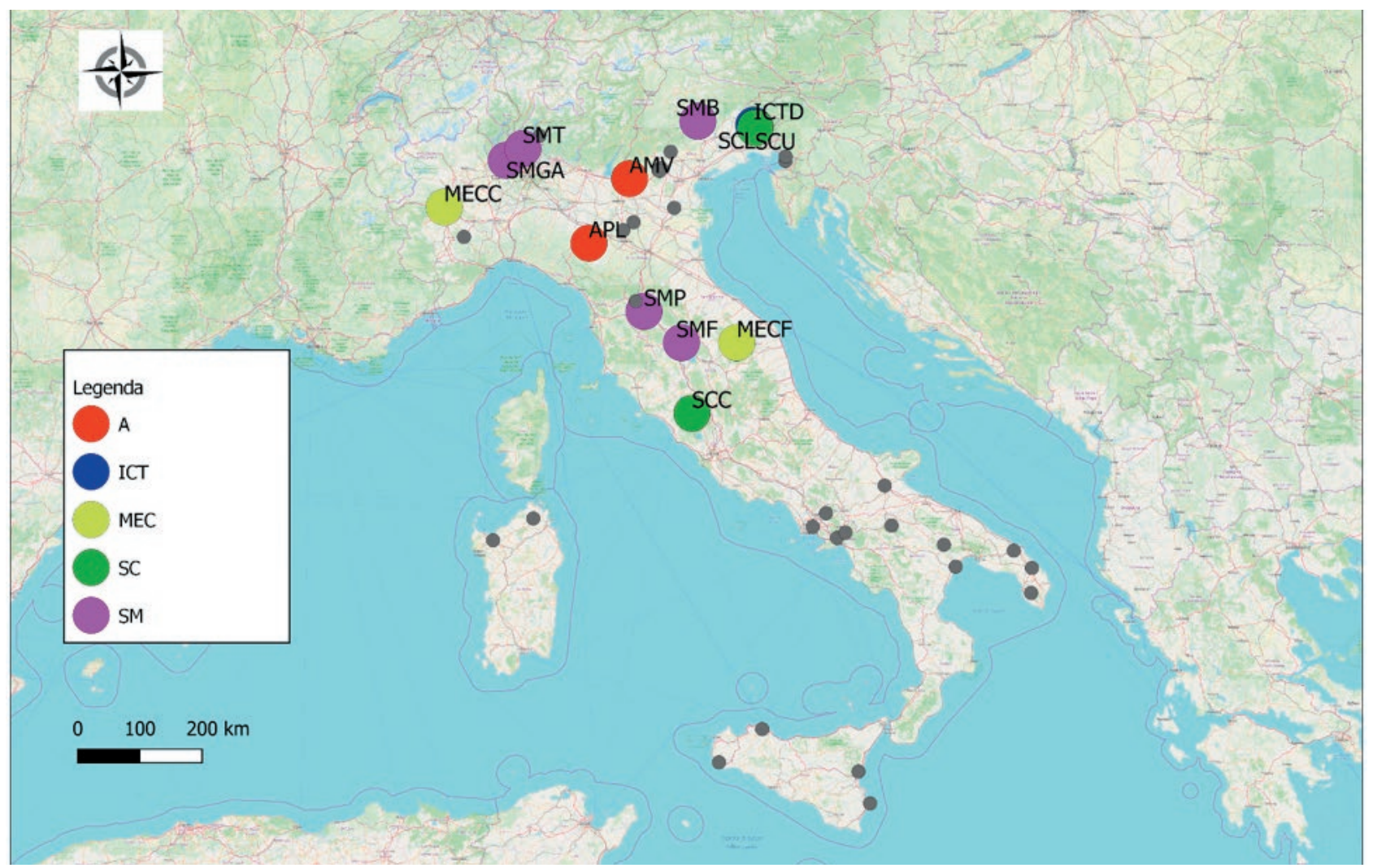

Figura 9b. Distribuzione geografia dei distretti del cluster PAS-B. Fonte: elaborazione dell'autore su dati Unioncamere. 


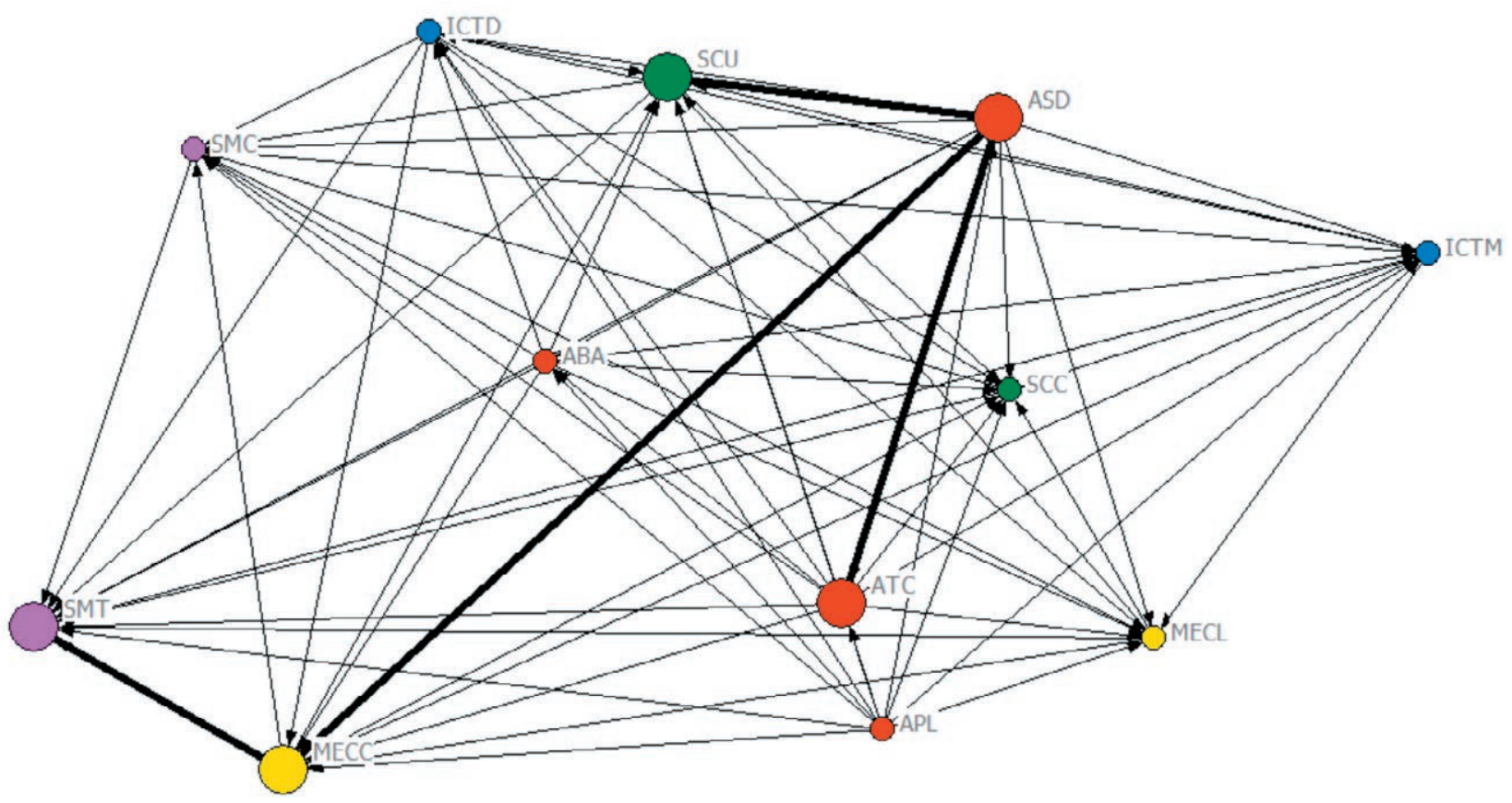

Figura 10a. Diagramma di clustering SRS-B con evidenza isomorfismi perfetti. Fonte: elaborazione dell'autore su dati Unioncamere con software Ucinet ${ }^{\star}$.

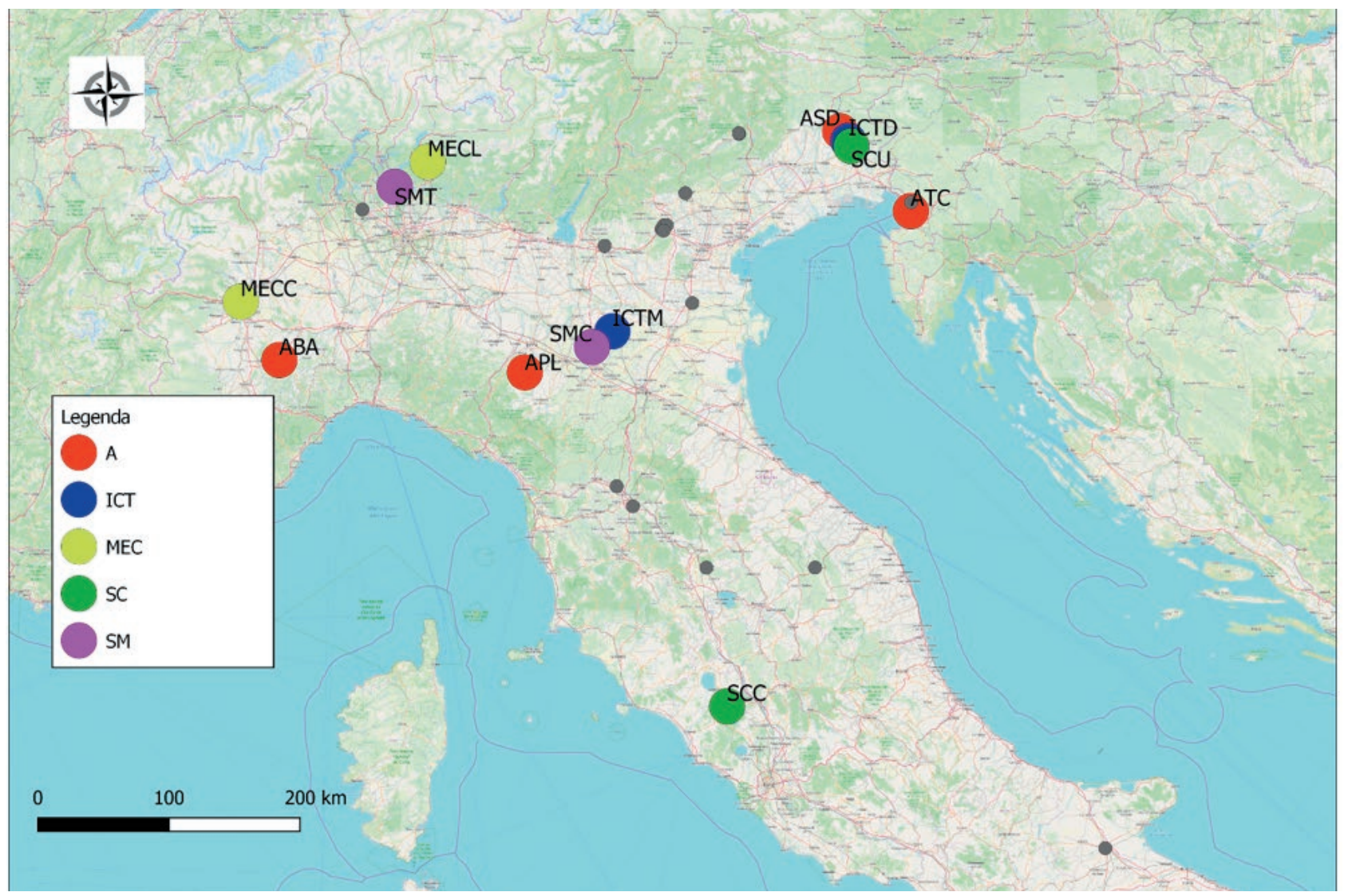

Figura 10b. Distribuzione geografia dei distretti del cluster SRS-B. Fonte: elaborazione dell'autore su dati Unioncamere. 


\subsection{Analisi dei risultati e discussione geografica}

L'analisi svolta mostra degli scenari non sempre convergenti in relazione alle variabili distrettuali considerate.

I cluster venutisi a formare secondo la prospettiva della variabile QIHT - Quota di Imprese ad Alta Tecnologia - mostrano una convergenza maggiore nella fascia alta della variabile rispetto a quelle medio-basse anche se evidenziano la minore capacità di clustering rispetto alle altre variabili (Tab. 1). Inoltre, palesano una prevalenza distributiva al Sud ed una prevalenza settoriale nei settori moda e agroalimentare. Si ravvisano anche due isomorfismi perfetti entrambi iso-settoriali, di cui uno Sud Sud non geograficamente prossimo, mentre l'altro in Sardegna caratterizzato da contiguità geografica.

Nell'ambito della fascia alta (Figg. 3a e 3b) di valori tra i distretti perfettamente isomorfi spiccano i distretti Calzature Napoletane e il Conciario di Solofra, che tuttora costituiscono ancora una realtà produttiva molto forte con un grosso peso sull'export. La Campania è, infatti, considerata una delle sette regioni calzaturiere italiane. In totale nella regione si contano, secondo dati Anci (2017), 432 aziende (il 7,4\% del totale nazionale) e gli addetti ammontano a 5.812 (il 7,3\%). L'85\% delle imprese non supera i 50 addetti e la tipologia di produzione, per effetto della concorrenza cinese sui prodotti di bassa gamma, si è spostata sulla linea di alta qualità. Questo è stato un ulteriore driver che ha spinto diverse aziende del settore moda ad abbandonare localizzazioni sparse o presenti nei centri storici delle città campane e a convergere nel distretto per incrementare i livelli di innovazione nella produzione, che conferma i dati profilati dalla variabile QIHT. Un esempio emblematico è la completa dismissione dell'area di Grumo Nevano (in provincia di Napoli) dove erano concentratele produzioni di bassa qualità ed una conseguente nuova localizzazione nell'area distrettuale delle imprese con maggiore vocazione alla qualità e alla innovazione che ne facevano parte. L'altro isomorfismo perfetto è quello dei Distretti di Calangianus e del Granito della Gallura, il primo regno del sughero estratto dalle querce che da millenni costituiscono una ricchezza ambientale ed economica di quell' area, ed il secondo specializzato nella lavorazione della pietra largamente impiegata sia nel settore dell'edilizia civile che nelle opere pubbliche.

Considerando il cluster di fascia bassa (Figg. 7a e 7b) si ottiene una situazione totalmente opposta alla precedente nella quale la distribuzione geografica vede una sola prevalenza del Nord.

La configurazione in cluster emersa attraverso la variabile SIHT Sviluppo Imprese ad Alta Tecnologia come nel caso precedente premia il Sud ma presenta una distribuzione geografica più omogenea. Si ravvisano diversi isomorfismi perfetti tutti intra-settoriali ma anche uno inter-settoriale tra i settori ICT e Sistema Moda, tutti localizzati al Sud e in prossimità geografica tra loro per la maggior parte. In questo caso, quindi, il dinamismo imprenditoriale segue criteri di contiguità geografica. L'analisi dei cluster operata in ordine a tale variabile rappresenta quella conseguente i maggiori valori in assoluto della variabile sia rispetto alle fasce della stessa variabile ma anche rispetto alle altre variabili (Tab. 1 e Figg. 4a e 4b). Quindi lo sviluppo di imprese ad alta tecnologia rappresenta il principale driver di affinità tra i distretti ed è su questa che andrebbe costruita una roadmap omogenea a livello Paese per puntare all'eccellenza.

L'analisi della fascia bassa della variabile induce un cluster distribuito esclusivamente nel Centro-Nord e sono ravvisabili diversi isomorfismi perfetti di tipo inter-settoriale soprattutto nel Nord-Est confermando il dinamismo imprenditoriale trasversale tipico dell'area (Figg. 78a e 8b). Dove sono, infatti, localizzati il distretto della Ceramica artistica e terracotta vicentina e il Metadistretto filiera Legno che hanno da tempo consolidato un patto di sviluppo, ravvisabile nelle linee programmatiche degli enti, basato sulla cooperazione di imprese operanti nel campo della lavorazione artistica della ceramica, della porcellana e del vetro, nonché di Enti pubblici e privati compartecipanti al progetto riuniti sotto un unico disegno strategico.

La variabile PAS - Popolazione ad Alta Scolarizzazione registra una maggiore capacità di clustering nella fascia bassa dei suoi valori. La condizione di isomorfismi perfetti è trasversalmente realizzata in fascia alta mediante convergenze inter-settoriali (Fig. 5a). La distribuzione geografica dei distretti in tale fascia (Fig. 5b) presenta concentrazioni al Sud e Nord senza una omogeneità diffusa che preveda anche il Centro.

Lo scenario relativo al cluster generato da valori di fascia bassa presenta tratti antitetici a quelli mostrati dal precedente caso relativo alla variabile QIHT - Quota di Imprese ad Alta Tecnologia, in quanto evidenzia una distribuzione prevalente del Sistema Moda nell'area del Centro Nord (Figg. 9a e 9b).

Infine, la variabile SRS - Spesa in Ricerca \& Sviluppo mostra in fascia alta una distribuzione geografica con polarizzazioni al Sud e ancora una volta con la prevalenza dei settori Moda e Agroalimentare e con diffusi isomorfismi intra-settoriali (Figg. 6a e 6b). Evidenza della propensione alla innovazione di tali settori in tali geografie.

Scenario antitetico, invece, quello di fascia bassa che vede una concentrazione al Nord molto varia nei settori, senza nessuna prevalenza particolare e con un nume- 

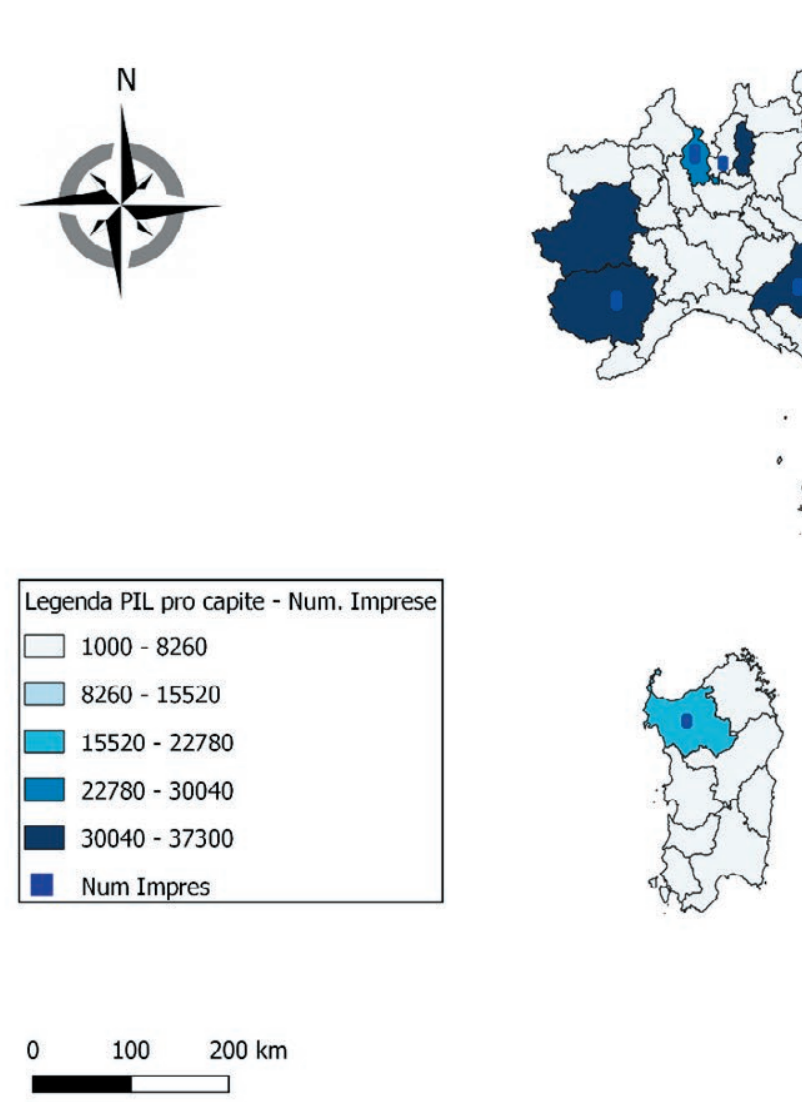

Figura 11. PIL pro capite e numero di imprese delle aree sede dei distretti. Fonte: elaborazione dellautore su dati Eurostat 2017.

ro minore di isomorfismi perfetti che denotano anche la presenza di gradienti nei valori.

Infine, appare interessante raccordare la specifica analisi condotta in ambito distrettuale con le condizioni territoriali al contorno delle aree distrettuali per verificare la presenza di eventuali altre correlazioni.

A tal fine, attingendo alle banche dati Eurostat ${ }^{8}$ sono state rilevate due variabili di contesto delle aree geografiche dei distretti analizzati, il PIL pro capite quale proxy della ricchezza del territorio di insediamento del distretto e il numero di imprese sorte nel periodo di riferimento quale indicatore del dinamismo imprenditoriale generale, da confrontare con quello specifico distrettuale indicato da una barra di istogramma sovrapposta (Fig. 11).

Dal punto di vista analitico è stata anche valutata sia l'analisi delle componenti principali ${ }^{9}$ (Appendice 3) e sia

\footnotetext{
${ }^{8}$ Employer business demography by NACE Rev. 2 and NUTS 3 regions [bd_enace2_r3]

${ }^{9}$ L'analisi delle componenti principali (APC) è una procedura statistica
}

la correlazione tra le 4 variabili prese in esame mediante una analisi di correlazione bivariata secondo BravaisPearson (Appendice 4). I risultati mostrano un duplice scenario. Il primo si contraddistingue per una prevalenza della prima variabile, Quota percentuale di Imprese high-tech, che, essendo una variabile di stock, evidenzia un carattere statico del fenomeno. Tale variabile risulta principale responsabile della variabilità dei dati e sottende un modello causale con le altre due variabili, Popolazione ad alta scolarità e Spesa in R\&S. Il secondo scenario, di tipo dinamico, vede la variabile di flusso Sviluppo imprese high-tech non correlata a nessuna di tali ultime due variabili.

atta a rilevare tra diverse variabili eventualmente correlate quella che consente di spigare maggiorente la varianza dei dati. L'APC trasforma matematicamente un insieme di variabili tra loro correlate in un numero minore di nuovi costrutti (detti componenti principali) indipendenti (cioè ortogonali fra loro nello spazio). Si tratta, quindi, sostanzialmente di una operazione che bilancia l'obiettivo della sintesi con quello di minimizzare la perdita di informazione. 
Dal punto di vista geografico tali evidenze analitiche, relative alla similitudine tra le ultime tre variabili, risultano ravvisabili nella presenza di un isomorfismo distrettuale nel Sud Italia riferito al Sistema Moda per i valori elevati di tali tre variabili, ed un isomorfismo distrettuale del Sistema Moda e del Sistema Casa nel Nord Italia per bassi valori delle tre variabili.

In conclusione, rispetto al test posto a base di analisi nella (2) è lecito, pertanto, affermare la sussistenza delle ipotesi $\mathrm{H}_{1}$ e $\mathrm{H}_{2}$ che fanno ritenere da un lato la metodologia di scouting di isomorfismi anche geograficamente non prossimi molto utile nella individuazione di convergenze relative a fattori strategici, e dall'altra che il fattore comune verso l'eccellenza distrettuale può anche prescindere entro certi limiti dai livelli del PIL del territorio insediativo ed essere totalmente orientato al dinamismo imprenditoriale e alla formazione di una forza lavoro avente una elevata scolarità.

\section{Conclusioni}

Una dicotomia spesso frequente nell'ambito degli studi geografici riguarda il dualismo tra fenomeni basati su modelli di propagazione e induzione inerenti variabili e fattori caratterizzanti aree contigue, e ricerche relative a omogeneità tra aree geograficamente non prossime. In questo secondo scenario rientrano, ad esempio, le città globali di Sassen unite in un circuito virtuale, caratterizzate da elementi simili slegati invece dal contesto geografico locale di riferimento.

Nel presente lavoro si è cercato di coniugare i due concetti in un unicum inteso come sistema-distrettuale a livello nazionale nel quale ogni nodo-distretto mantiene le sue caratteristiche intrinseche valoriali legate alla comunità locale, ma allo stesso tempo nell'ambito del sistema è parte di uno o più cluster. Tale finalità è stata perseguita proponendo un approccio basato su un impiego differente dell'efficace background relativo alla teoria dei grafi.

In particolare, il modello sviluppato configura l'insieme dei distretti industriali italiani come i nodi di una rete il cui grado di "magliatura", che descrive l'architettura dei rami di collegamento tra i nodi stessi, risulta definito a partire da condizioni di prossimità non geografica ma relativa all'affinità tra alcuni attributi espressi da variabili distrettuali presente nella sorgente dati analizzata. Si è inteso quindi riferirsi a tale scenario in termini di isomorfismi, ossia relazioni codificate nella teoria matematica che ben si prestavano agli obiettivi di ricerca.

La condizione generativa dei cluster è la consistenza di relazioni che in questo caso sono intese quali relazioni di convergenza di alcune variabili inerenti ai paradigmi della innovazione distrettuale.

La valenza dell'approccio proposto è di duplice natura, riguardando da un lato lo specifico ambito distrettuale sia con implicazioni scientifiche che pratiche, dall'altro una eventuale trasponibilità del metodo ad ambiti differenti.

Una prima esternalità positiva del modello sviluppato attiene ad una ulteriore prospettiva di analisi, rispetto alle numerose e consolidate presenti in letteratura, che viene fornita in relazione al fenomeno distrettuale e alle relative dinamiche. Una prospettiva orientata alla scala nazionale e basata sulla comparazione delle variabili distrettuali e sulla ricerca della eventuale influenza di determinismi geografici sui valori che esse assumono.

Con riguardo a tale aspetto dall'analisi è emersa una rilevante influenza del dinamismo imprenditoriale del luogo rispetto alla efficacia produttiva del distretto, come già sostenuto da diversi autori (ad esempio, OwenSmith e Powell, 2006) ed una diffusa presenza di cluster isomorfi geograficamente non prossimi sia di tipo intersettoriale che intra-settoriale.

Dal punto di vista delle implicazioni pratiche che possono derivare dal lavoro svolto, la disponibilità delle stratificazioni operate atte a evidenziare reti di distretti isomorfi può consentire a manager di impresa, rappresentanti di associazioni di categoria, e in generale agli addetti ai lavori operanti nell'ambito dei distretti, di identificare eventuali strategie comuni per l'eccellenza laddove i distretti condividano un posizionamento in fascia elevata di una delle variabili rilevate e, viceversa, azioni sinergiche per il miglioramento laddove, invece, si ravvisi una convergenza verso i valori più bassi di qualcuna delle variabili.

La seconda esternalità derivante dalla ricerca esula dall'ambito distrettuale e riguarda eventuali positivi effetti di trasferibilità dell'approccio seguito. Tale lavoro ambisce, infatti, a fornire una metodologia replicabile in scenari analoghi nei quali risulti interessante valutare analiticamente la similitudine tra aree geograficamente non prossime. In altri termini, si ritiene che l'utilità di tale modello risieda nella sua trasversalità di applicazione ai numerosi scenari nei quali la condivisione di strategie comuni possa risultare un valore aggiunto per la co-creazione di valore.

Un esempio in tal senso è ravvisabile nel rischio ambientale che, storicamente, si caratterizza per approcci di prossimità nella governance emergenziale, laddove invece potrebbe risultare conveniente identificare reti di aree geograficamente non prossime, equivalenti ai distretti analizzati, soggette a stessi fattori di 
rischio di intensità variabile da valori bassi a valori elevati come nel caso trattato.

Un altro esempio può riguardare la promozione e la valorizzazione delle azioni di tutela della proprietà intellettuale, in particolare con riferimento ai brevetti, che potrebbe essere basata su una conveniente identificazione di aree non contigue nelle quali è ravvisabile una medesima propensione alla brevettazione.

Molti altri esempi di fenomeni geograficamente diffusi a macchia di leopardo rappresentativi di variabili in comune potrebbero, pertanto, beneficiare dell'approccio proposto.

Tra i limiti della ricerca, due aspetti necessitano di evidenza.

Il primo attiene alla fonte dei dati impiegati. La ricerca si è proposta di indagare una categoria geoeconomica, il distretto industriale, attraverso indicatori provinciali o regionali, quando tipicamente il distretto è un'entità funzionale sub-regionale, sub-provinciale, non infrequentemente trans-provinciale, e in ogni caso costituita da aggregati di comuni profondamente differenti dalle normali partizioni amministrative. La scelta di utilizzare questi dati è derivata, tuttavia, dall'indisponibilità dei medesimi a scala comunale.

Pertanto, l'approccio proposto, nella consapevolezza della impossibilità a presentare risultati solidamente fondati e univocamente riconducibili all'unità territoriale del distretto, è risultato animato dalla volontà di presentare una proposta interpretativa basata su un impianto metodologico, di utilità implementativa laddove siano disponibili dati alla scala più idonea.

Il secondo aspetto riguarda l'opportunità di incrementare il rango di indagine, in quanto l'analisi proposta, anche per esigenze di contenimento in un articolo, è stata condotta in modalità OFAT (One Factor At Time), fermandosi cioè alla condizione di "almeno una variabile" in comune tra i distretti quale driver di innesco dello stato distrettuale isomorfo generativo del cluster. Un contributo incrementale di informazione in un follow up della ricerca può, infatti, derivare da un approccio multi-variabile simultaneo, nel quale approfondire anche le interazioni intra-variabili.

\section{Ringraziamenti}

L'autore desidera ringraziare i revisori che hanno consentito di migliorare notevolmente il presente contributo con un dettaglio analitico di modifiche e suggerimenti proposti che dimostra una grande dedizione e attenzione posta al lavoro che hanno esaminato.

\section{Riferimenti bibliografici}

Balland, P.A., Boschma, R., Frenken, K. (2015). Proximity and Innovation: From Statics to Dynamics. Regional Studies, 49 (6), 907-920.

Becattini, G. (a cura di) (1969). Lo sviluppo economico della Toscana: un'ipotesi di lavoro. Firenze, IRPET.

Becattini, G. (a cura di) (1975). Lo sviluppo economico della Toscana. Firenze, IRPET.

Becattini, G. (1990). The Marshallian industrial district as a socio-economic notion. In Pyke, F., Becattini, G., Sengenberger, G. (eds.), Industrial districts and inter-firm co-operation in Italy. Geneva, International Institute for Labour Studies, 37-51.

Becattini, G., Bellandi, M., de Propris, L. (2009). Critical nodes and contemporary reflections on industrial districts: An introduction. In Becattini, G., Bellandi, M., de Propris, L. (eds.), A handbook on industrial districts. Cheltenham, Edward Elgar, $\mathrm{xv}-\mathrm{xxxv}$.

Becattini, G., Rullani E. (1993). La natura e la dinamica dell'impresa distrettuale. Economia e Politica Industriale, 80, 25-48.

Bell, G.G. (2005). Clusters, Networks, and Firm Innovativeness. Strategic Management Journal, 26 (3), 287 - 295, DOI: $10.1002 / \mathrm{smj} .448$.

Belussi, F. (2015). The international resilience of Italian industrial districts/clusters between knowledge re-shoring and manufacturing off (near)-shoring. Investigaciones Regionales, 32, 89-113.

Bonavero, P. (1995). I contesti locali di eccellenza tecnologica: concetti e strumenti per l'analisi dei sistemi innovativi. Geotema, 2, 68-85.

Brusco, S. (1975). Economie di scala a livello tecnologico nelle piccole imprese. In Graziani, A. (a cura di), Crisi e ristrutturazione nelleconomia italiana, Torino, Einaudi.

Brusco, S. (1989). Piccole imprese e distretti industriali una raccolta di saggi. Torino, Rosenberg \& Sellier.

Camison, C., Molina-Morales, F.X. (1998). El distrito industrial cerámico valenciano: mito o realidad competitiva? Revista Valenciana D'Estudis Autonòmics, 22, 83-102.

Colavitti, A.M., Usai, A. (2020). Inside the system-wide cultural district: a new relational and organisational taxonomy of cultural districts based on the sector policies by Italian Regions (2000-2015). City, Territory and Architecture 7 (4), 67-78. DOI: 10.1186/s40410-020-0112-1 
Conti, S. (2012). I territori delleconomia. Fondamenti di geografia economica. Torino, Utet.

Cortés, E.C., Lajara, B.M., Larrosa, P.S., Fernández L.R. (2019). Competitive advantage and industrial district. International Business Journal, 29 (3), 211-235. DOI:10.1108/CR-08-2018-0048

Dini, F. (1995). Riflessioni sulle economie di agglomerazione come nesso materiale e immateriale della territorialità dell'industria. Geotema, 2, 22-30.

Falorni, A. (2013). Sistemi locali ed imprese: unianalisi dello scenario evolutivo italiano. Firenze, Firenze University Press.

Feld, S. L. (1981). The focused organization of social ties. American Journal of Sociology, 86, 1015-1035.

Festa, G., Rossi, M., Kolte, A., Situm, M. (2020). Territory-based knowledge management in international marketing processes - the case of "Made in Italy" SMEs". European Business Review, 32 (3), 435-442. DOI: 10.1108/ EBR-06-2019-0129

Fuensanta, M.J.R. (2010). Influencia de las economías externas de distrito sobre la productividad empresarial: Un enfoque multinivel", Investigaciones Regionales. Journal of Regional Research, 18, 61-82.

Heider, F. (1946). Attitudes and cognitive organization. Journal of Psychology, 21, 107-112.

Holland, P. W., Leinhardt, S. (1970). A method for detecting structure in sociometric data. American Journal of Sociology, 76, 492-513.

Holland, P. W., Leinhardt, S. (1971). Transitivity in structural models of small groups. Comparative Group Studies, 2, 107-124.

Hofstadter, D. (1979). Gödel, Escher, Bach: un'eterna ghirlanda brillante. New York, Basic Books.

Konzelmann, S., Wilkinson, F., Fovargue-Davies, M. (2016). Britain's Industrial Evolution: From Industrial Districts to Large Scale Production and Back Again, paper presented at the 40 Years of the Cambridge Journal of Economics Conference, Cambridge, 12-13 July

ISTAT (2015). I distretti industriali 2011. $9^{\circ}$ Censimento dell'industria e dei servizi e Censimento delle istituzioni non profit. Roma.

Las Casas G., Scardaccione G., Scorza F. (2009). Metodi geostatistici per la clusterizzazione dei flussi migratori in Italia. Atti 13-ma Conferenza Internazionale Asita Bari 1-4 dicembre 2009.
Louch, H. (2000). Personal network integration: Transitivity and homophily in strong-tie relations. Social Networ$k s, 22,45-64$.

Luce, R. D., Perry, A.D. (1949). A method of matrix analysis of group structure. Psychometrika, 14, (1), 95-116. DOI:10.1007/BF02289146

Mariotti, I., Barzotto, M., Corò, G. (2020). Industrial districts, urban areas or both? The location behaviour of foreign and domestic firms in an Italian manufacturing region. The Annals of Regional Science, 64, 523-546. DOI: 10.1007/s00168-020-00990-8

Marshall, A. (1920). Principles of Economics, $8^{\text {th }}$ ed. London, Macmillan.

Marshall, A. (1923). Industry and Trade, $4^{\text {th }}$ ed. London, Macmillan.

Owen-Smith, J., Powell, W.W. (2006). Accounting for emergence and novelty. In Braunerhjelm, P., Feldman, M. (eds.), Boston and Bay Area biotechnology in cluster genesis: Technology-based industrial development. Boston, Harvard Business School Press, 61-86.

Padgett, W.W., Powell J. (2012). The Emergence of Organizations and Markets. Princeton-Oxford, Princeton University Press.

Puig, F., Marques, H. (2011). The Dynamic Evolution of the Proximity Effect in the Textile Industry, European Planning Studies, 19 (8), 1423-1439. DOI: 10.1080/09654313.2011.586174

Randelli, F., Rocchi B., Stefania G. (2017). Alternative food networks e città in Italia: un'analisi spaziale a partire dai dati di censimento. Bollettino della Società Geografica Italiana, serie 13, 10 (1-2), 165-175. DOI: 10.13128/bsgi. v10i1-2.505

Rullani, E. (2002). Il distretto industriale come sistema complesso adattivo. In Quadro Curzio, A., Fortis M. (a cura di), Complessità e distretti industriali. Dinamiche, modelli, casi reali. Bologna, Il Mulino, 65-107

Sforzi, F. (2008). The Industrial District: From Marshall to Becattini. Il pensiero economico italiano, 16 (2), 71-80.

Sforzi, F. (2014). The empirical evidence of industrial districts in Italy. In Becattini, G., Bellandi, M., de Propris, L. (eds.), A handbook on industrial districts. Cheltenham, Edward Elgar, 327-333.

Simboli, A., Taddeo, R., Raggi, A., Morgante, A. (2020). Structure and Relationships of Existing Networks in View of the Potential Industrial Symbiosis Development. In Salomone R., Cecchin, A., Deutz, P., Raggi, A., Cutaia, L. 
(eds), Industrial Symbiosis for the Circular Economy. Strategies for Sustainability. Cham, Springer.

Snijders, T.A.B. (2001). The statistical evaluation of social network dynamics. Sociological Methodology, 31, 361-395.

Snijders, T.A.B., Pattison, P.E., Robins, G.L., Handcock, M.S. (2006). New specifications for exponential random graph models. Sociological Methodology, 35, 99-153.

Taylor, M. (1995). Impresa industriale, relazioni di potere e modelli di industrializzazione geografica. Geotema, 2, 9-21.

Tinacci Mossello, M. (1982). Economia e geografia. Dall'analisi delle economie di agglomerazione alla teoria dello sviluppo regionale. Rivista Geografica Italiana, 2, 303-333.

Tödtling, F. (1995). Processi di innovazione, imprese e ambiente locale. Geotema, 2, 42-53.

Watts, D.J., Strogatz, S.H. (1998). Collective dynamics of "small-world" networks. Nature, 393, 440-442. 


\section{Appendici}

Appendice 1. Base dati distretti analizzati. Fonte: elaborazione dellautore per estrapolazione dai dati Unioncamere.

\begin{tabular}{|c|c|c|c|c|c|}
\hline ID & Denominazione & $\begin{array}{l}\text { Quota Imprese } \\
\text { high-tech (\%) }\end{array}$ & $\begin{array}{c}\text { Sviluppo imprese } \\
\text { high-tech (\%) }\end{array}$ & $\begin{array}{l}\text { Popolazione ad alta } \\
\text { scolarità }(\%)\end{array}$ & Spesa R\&S (\%) \\
\hline APL & Agroalimentare di Parma-Langhirano & 11 & 89 & 9 & 13 \\
\hline AMV & Metadistretto alimentare Veneto & 40 & 18 & 21 & 56 \\
\hline ASD & Agroalimentare di San Daniele & 41 & 53 & 31 & 19 \\
\hline ATC & Trieste Coffee Cluster & 9 & 98 & 26 & 19 \\
\hline $\mathrm{ABA}$ & Bevande Alcooliche Canelli Alba & 89 & 40 & 78 & 2 \\
\hline AIR & Ittico di Rovigo & 94 & 11 & 97 & 56 \\
\hline AVU & Agroalimentare del Vulture & 88 & 94 & 70 & 98 \\
\hline ANI & Agroalimentare Nocera Inferiore & 79 & 49 & 68 & 50 \\
\hline API & Co.S.Va-P. Pesca Industriale & 95 & 21 & 93 & 80 \\
\hline AME & Metapontino Agroalimentare & 96 & 69 & 75 & 98 \\
\hline ICTM & Biomedicale di Mirandola & 13 & 8 & 44 & 13 \\
\hline ICTD & Tecnologie Digitali DITEDI & 41 & 53 & 31 & 19 \\
\hline ICTT & Nautica, ICT, Biotecnologie Triestino & 9 & 98 & 26 & 19 \\
\hline ICTA & Aerospaziale Pugliese & 100 & 81 & 100 & 84 \\
\hline MECL & Metalli Lecchese & 7 & 85 & 59 & 27 \\
\hline MECV & Meccatronica Vicentina & 3 & 43 & 55 & 56 \\
\hline MECC & Componentistica e della Meccanica (Co.Mec) & 38 & 41 & 23 & 19 \\
\hline MECF & Meccanica ed Elettrodomestici di Fabriano & 49 & 90 & 19 & 94 \\
\hline MECP & Meccanica Pugliese & 84 & 73 & 88 & 84 \\
\hline MECS & Meccanica Siciliana & 85 & 47 & 69 & 80 \\
\hline MECPA & Meccatronica di Palermo & 93 & 49 & 90 & 80 \\
\hline SCBA & Mobile d'arte di Bassano & 3 & 43 & 55 & 56 \\
\hline SCV & Ceramica artistica e Terracotta vicentina & 8 & 32 & 52 & 56 \\
\hline SCL & Metadistretto filiera Legno (Veneto e Friuli V.G.) & 36 & 37 & 19 & 55 \\
\hline SCB & Energie Rinnovabili Belluno & 48 & 2 & 46 & 56 \\
\hline SCU & Sedia Udinese & 41 & 53 & 31 & 19 \\
\hline SCC & Ceramica di Civica Castellana & 101 & 101 & 22 & 9 \\
\hline SCM & Mobile imbottito dell'Area Murgiana & 80 & 61 & 83 & 89 \\
\hline SCS & Sughero di Calangianus-Tempio Pausania & 86 & 57 & 95 & 100 \\
\hline SCG & Granito della Gallura & 86 & 57 & 95 & 100 \\
\hline SMC & Tessile-Abbigliamento di Carpi & 13 & 8 & 44 & 13 \\
\hline SMGA & Abbigliamento Gallaratese & 2 & 88 & 17 & 27 \\
\hline SMV & Orafo-Argentiero di Vicenza & 3 & 43 & 55 & 56 \\
\hline SMCV & Concia Vicentina & 3 & 43 & 55 & 56 \\
\hline SMB & Occhiale Bellunese & 39 & 5 & 39 & 56 \\
\hline SMT & Tessile e Abbigliamento di Como & 37 & 95 & 36 & 27 \\
\hline SMF & Pelle e Cuoio Castiglion Fiorentino & 44 & 30 & 18 & 40 \\
\hline SMP & Pelle e Cuoio Valdarno Superiore & 46 & 16 & 15 & 40 \\
\hline SMPR & Tessile Abbigliamento di Prato & 45 & 26 & 41 & 39 \\
\hline SMPU & Produttivo Fiera Moda Puglia & 92 & 78 & 89 & 84 \\
\hline SMN & Calzature Napoletane & 72 & 93 & 94 & 50 \\
\hline SMS & Conciario di Solofra & 98 & 87 & 72 & 50 \\
\hline SMG & Tessile S. Agata dei Goti & 97 & 83 & 51 & 50 \\
\hline SMSO & Tessile Sicilia Orientale & 89 & 82 & 86 & 80 \\
\hline SML & Calzature di Casarano-Lecce & 98 & 84 & 91 & 84 \\
\hline
\end{tabular}


Appendice 2. Curva di errore. Fonte: elaborazione dell'autore con il software EffeDiX.

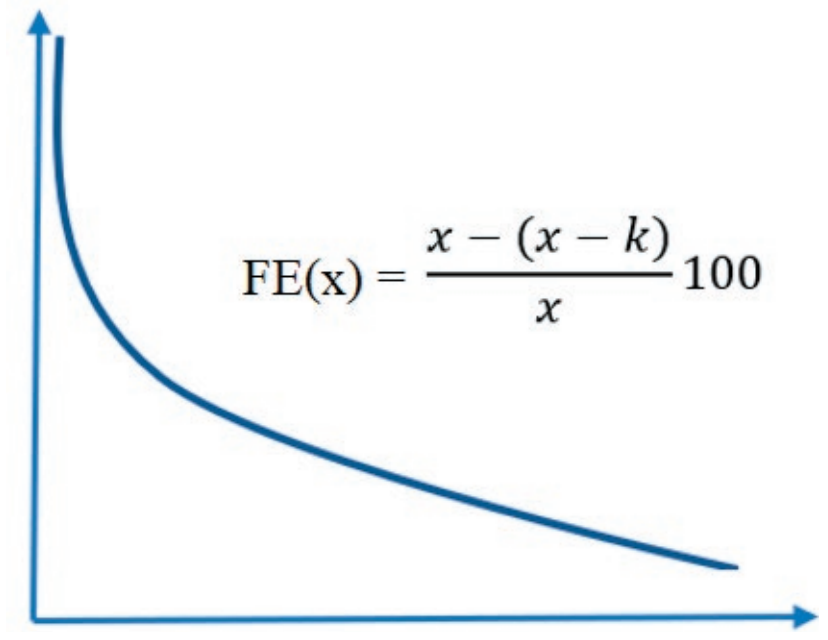

Appendice 3. Analisi delle componenti principali

Data variables:

QIHT

SIHT

PAS

SRS

Data input: observations

Number of complete cases: 45

Missing value treatment: listwise

Standardized: yes

Number of components extracted: 1

Principal Components Analysis

\begin{tabular}{cccc}
\hline $\begin{array}{c}\text { Component } \\
\text { Number }\end{array}$ & Eigenvalue & $\begin{array}{c}\text { Percent of } \\
\text { Variance }\end{array}$ & $\begin{array}{c}\text { Cumulative } \\
\text { Percentage }\end{array}$ \\
\hline 1 & $(2,257)$ & $(56,436)$ & $(56,436)$ \\
2 & 0,985 & 24,649 & 81,086 \\
3 & 0,455 & 11,389 & 92,474 \\
4 & 0,301 & 7,526 & 100,000 \\
\hline
\end{tabular}

\section{The StatAdvisor Principal Component}

One component has been extracted, since only one component had an eigenvalue greater than or equal to 1,0 . It accounts for $56,4361 \%$ of the variability in the original data.

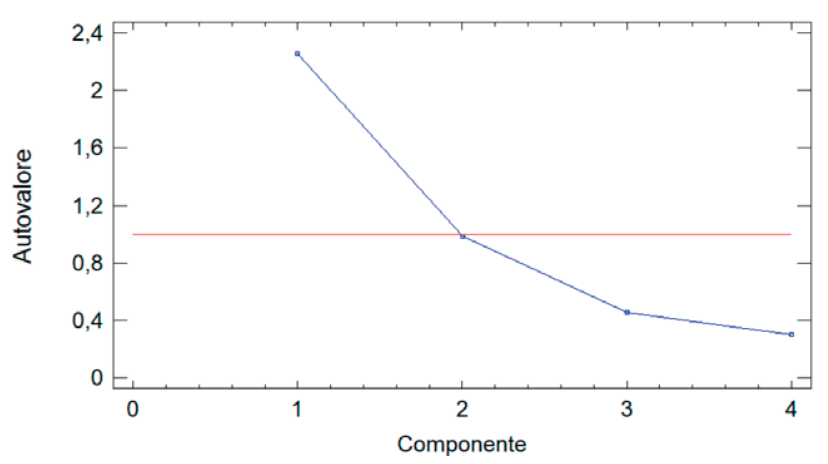

Appendice 4. Matrice di correlazione bivariata. Fonte: elaborazione dell'autore su dati Unioncamere.

\begin{tabular}{ccccc}
\hline & $\begin{array}{c}\text { Quota } \\
\text { Imprese } \\
\text { high-tech }\end{array}$ & $\begin{array}{c}\text { Sviluppo } \\
\text { imprese } \\
\text { high-tech }\end{array}$ & $\begin{array}{c}\text { Popolazione } \\
\text { ad alta } \\
\text { scolarità }\end{array}$ & Spesa R\&S \\
\hline $\begin{array}{c}\text { Quota } \\
\text { Imprese }\end{array}$ & 1 & & & \\
high-tech \\
$\begin{array}{c}\text { Sviluppo } \\
\text { imprese } \\
\text { high-tech }\end{array}$ & 0,198 & 1 & & \\
$\begin{array}{c}\text { Popolazione } \\
\text { ad alta }\end{array}$ & $(0,656)$ & 0,072 & 1 & \\
scolarità \\
Spesa R\&S & $(0,533)$ & 0,079 & $(0,646)$ & 1 \\
\hline
\end{tabular}

